\title{
Biased orientation representations can be explained by experience with non- uniform training set statistics
}

Margaret Henderson ${ }^{1}$, John Serences ${ }^{1,2,3}$

${ }^{1}$ Neurosciences Graduate Program, University of California, San Diego, La Jolla, California 92093-1090 USA

${ }^{2}$ Department of Psychology, University of California, San Diego, La Jolla, California 92093-0109 USA

${ }^{3}$ Kavli Foundation for the Brain and Mind, University of California, San Diego, La Jolla, California 92093 USA

\section{Author Contributions:}

All authors conceived the experiments and edited the manuscript. $\mathrm{MH}$ implemented the experiments and analysis and wrote the manuscript. JTS supervised the project.

\section{Correspondence:}

Margaret Henderson

Neurosciences Graduate Program

University of California, San Diego

9500 Gilman Drive, La Jolla, CA, 92093

mmhender@ucsd.edu

Authors report no conflict of interest. 


\section{Abstract}

3 Visual acuity is better for vertical and horizontal compared to other orientations. This cross-

4 species phenomenon is often explained by "efficient coding", whereby more neurons show

5 sharper tuning for the orientations most common in natural vision. However, it is unclear if

6 experience alone can account for such biases. Here, we measured orientation representations in

7 a convolutional neural network, VGG-16, trained on modified versions of ImageNet (rotated by

$80^{\circ}, 22.5^{\circ}$, or $45^{\circ}$ counter-clockwise of upright). Discriminability for each model was highest near

9 the orientations that were most common in the network's training set. Furthermore, there was

10 an over-representation of narrowly tuned units selective for the most common orientations.

11 These effects emerged in middle layers and increased with depth in the network. Biases emerged

12 early in training, consistent with the possibility that non-uniform representations may play a

13 functional role in the network's task performance. Together, our results suggest that biased

14 orientation representations can emerge through experience with a non-uniform distribution of

15 orientations, supporting the efficient coding hypothesis.

\section{Keywords}

20 Orientation, perceptual bias, anisotropy, efficient coding, convolutional neural network 


\section{Introduction}

25 Contrary to common intuition, visual perception is not perfectly uniform across orientation

26 space. One example of this principle is the "oblique effect", which has been demonstrated in

27 humans and a wide range of animal species, including cats, octopi and goldfish, among others.

28 This effect describes the finding that observers' ability to discriminate small changes in

29 orientation, as well as other forms of acuity, tend to be worst for stimuli that have edges

30 oriented diagonally (oblique orientations) and better for stimuli with edges oriented vertically

31 or horizontally (cardinal orientations) (Appelle, 1972; Bauer, Owens, Thomas, \& Held, 1979). In

32 visual cortex, this finding has been linked to a larger number of orientation tuned neurons with

33 a preference for cardinal orientations, as has been shown in cats (Li, Peterson, \& Freeman,

34 2003), and macaques (Mansfield, 1974; Shen et al., 2014), among other species. Some evidence

35 also suggests that cardinally-tuned neurons may have narrower tuning than other orientations,

36 which may also contribute to higher acuity (Kreile, Bonhoeffer, \& Hübener, 2011; Li et al.,

37 2003).

39 One compelling explanation for the origin of the oblique effect is the efficient coding

40 hypothesis, which suggests that because the brain operates with limited resources, coding

41 resources should be preferentially allocated to stimuli that are highly probable during natural

42 vision (Barlow, 1961; Girshick, Landy, \& Simoncelli, 2011). On this view, biased orientation

43 perception may reflect an adaptation to the statistics of natural images, in which vertical and

44 horizontal orientations are most common (Coppola, Purves, McCoy, \& Purves, 1998; Girshick et 
45 al., 2011). Support for an experience-driven account of the oblique effect includes evidence

46 that in primates, the over-representation of cardinal orientations in visual cortex increases with

47 age (Shen et al., 2014). Additionally, exposing developing kittens or mice to an environment

48 with contours of only one orientation can induce changes in the distribution of cortical

49 orientation tuning, suggesting some degree of plasticity (Blakemore \& Cooper, 1970; Hirsch \&

50 Spinelli, 1970; Kreile et al., 2011; Leventhal \& Hirsch, 1975). Finally, past computational work

51 demonstrates that various models of optimal information coding, along with measurements of

52 environmental statistics, can be used to predict both the neural and behavioral correlates of

53 the oblique effect (Ganguli \& Simoncelli, 2011; Girshick et al., 2011; Wainwright, 1999).

55 In addition, innate factors may also contribute to the efficient coding of cardinal orientation.

56 For instance, while it is possible to significantly modify the distribution of orientation tuning

57 preferences in visual cortex through experience, exposing an animal to only diagonal lines

58 during development does not entirely obliterate tuning for cardinal orientations (Kreile et al.,

59 2011; Leventhal \& Hirsch, 1975). Similarly, rearing animals in complete darkness can result in a

60 more extreme over-representation of cardinal-tuned units (Leventhal \& Hirsch, 1980). In both

61 mice and ferrets, it has been suggested that innate factors result in a strong oblique effect early

62 in development, while visual experience tends to make orientation tuning more uniform over

63 time (Coppola \& White, 2004; Hoy \& Niell, 2015). These observations are consistent with the

64 efficient coding account if we assume that the visual system can adapt to environmental

65 regularities over the course of evolution, resulting in feature biases that are encoded in the 66 genome. 
68 However, factors that are independent of visual input statistics may also separately contribute

69 to the presence of cardinal orientation biases in animals. For example, some anatomical

70 properties of the visual system naturally give a privileged status to the cardinal axes, such as

71 the horizontal raphe of the retina, the role of the horizontal axis in vestibular and oculomotor

72 system organization, and the distinction between processing of vertical and horizontal disparity

73 (Westheimer, 2003). Such properties need not be related to the orientation content of natural

74 images, but may instead reflect general physical and/or developmental constraints. It is

75 plausible that the presence of these architectural factors leads to cardinal biases, independent

76 from the statistics of natural images. Thus, while past computational work suggests a strong

77 correlational link between the statistics of the environment and the representation of

78 orientation, the causal link between these observations has not yet been established.

79 Here, we use a causal manipulation to evaluate whether the efficient coding mechanism alone

80 can account for the emergence of the oblique effect.

82 Specifically, we achieved this by measuring orientation representations in a convolutional

83 neural network (CNN). We focus on the popular VGG-16 model, a standard feedforward

84 network that achieves high performance at classifying objects in natural images (Simonyan \&

85 Zisserman, 2014). We first test whether a pre-trained VGG-16 model exhibits the classical

86 oblique effect, assessed using the Fisher information measured at entire layers of the network,

87 and the distribution of single-unit tuning properties. Assuming a definition of efficient coding in

88 which mutual information between the stimulus and the network's responses is maximized, we 
89 predict that Fisher information will be proportional to the square of the prior distribution

90 (Figure 1D; Ganguli \& Simoncelli, 2011; Wei \& Stocker, 2015). In addition to a test of the

91 efficient coding hypothesis, measuring orientation bias in this pre-trained model will provide an

92 assessment of whether existing CNNs, often used as models of the primate visual system (Cichy

93 \& Kaiser, 2019; Kell \& McDermott, 2019), exhibit this defining characteristic of biological vision.

95 We next trained VGG-16 models on modified versions of the ImageNet database (Deng et al.,

$962009)$ that had been rotated by $0^{\circ}, 22.5^{\circ}$ or $45^{\circ}$ relative to upright. This allowed us to

97 determine whether a bias centered around other axes can be induced equally as well as a

98 cardinal bias, and whether the biases observed in the pre-trained network were simply artifacts

99 of some intrinsic property of the CNN (e.g. a square pixel grid that results in a cardinal

100 reference frame). We demonstrate that, contrary to this alternative, networks trained on

101 rotated images exhibited rotated biases that were consistent with the networks' training set

102 statistics. These results suggest that general visual experience with a non-uniform orientation

103 distribution is sufficient to promote the formation of biased orientation representations.

104 Further, our findings highlight how biased training data can fundamentally impact visual

105 information processing in neural network models.

106

107 Materials and Methods

108

109

\section{Training stimuli}


111 During training, each model was presented with a modified version of the ILSVRC-2012-CLS

112 training image set, a set of $\sim 1.3$ million colored images with substantial variability in layout and

113 background, each including an object in one of 1,000 categories (Deng et al., 2009; Russakovsky

114 et al., 2015). Three modified versions of this image set were generated, corresponding to

115 rotations of $0^{\circ}, 22.5^{\circ}$, and $45^{\circ}$ counter-clockwise relative to vertical. The purpose of generating

$116 \mathrm{a} 0^{\circ}$ (no rotation) version of the image set was to provide a control to isolate the effect of

117 image rotation from any other properties of our modified image set.

119 To generate each version of the image set, we loaded each image from the original ILSVRC

120 image set, rotated it by the specified amount, and cropped the image centrally by a specified

121 amount that was the same for all rotations. Images were then scaled to a size of [224 x 224]

122 pixels, and multiplied by a smoothed circular mask. The smoothed mask set to background all

123 pixels at a radius of more than 100 pixels from the center, retained all pixels at a radius of less

124 than 50 pixels from the center, and applied a cosine function to fade out the intermediate

125 pixels. Finally, the background pixels were adjusted to a grey color that closely matches the

126 mean RGB value of the training ImageNet images (Simonyan \& Zisserman, 2014). All image

127 processing for training set images was done in Python 3.6 (Python Software Foundation,

128 Wilmington DE) using the Python Imaging Library. For each training set, a corresponding

129 validation set was generated using the same procedure, and this validation set was used to

130 evaluate performance during training. When preprocessing the images for training and

131 validation, we modified the procedure from Simonyan and Zisserman's paper by skipping the 
132 random rescaling and random left-right flipping steps. The purpose of this was to preserve the

133 original spatial frequency and orientation content of the images as closely as possible.

\section{Evaluation stimuli}

137 Networks were evaluated using sets of images that had known orientation content but were 138 variable in their spatial phase and frequency (Figure 1B). These images consisted of randomly 139 sampled images from the ILSRVC-2012-CLS image set which were filtered to have a particular 140 orientation content. Before filtering each image, we first rotated it by a randomly chosen value

141 in the range of 0-179 degrees, then cropped it centrally to a square and scaled to a size of [224

$142 \times 224]$ as described above. This was done to prevent any dependencies between orientation

143 and other low-level image properties, such as spatial frequency content and luminance

144 contrast, in the final filtered images. After this step, we converted to grayscale, z-scored the

145 resulting luminance values, and masked the image with the smoothed circular mask described

146 above. The image was then padded with zeros to a size of [1012 x 1012] pixels, and

147 transformed into the frequency domain (using fft2. $m$ ). We then multiplied the frequency-

148 domain representation by an orientation filter and a spatial frequency filter. The orientation

149 filter consisted of a circular Gaussian (Von Mises) function centered at the desired orientation,

150 with concentration parameter $(k)$ of 35 (full-width at half-max $=11.5^{\circ}$ ). The spatial frequency

151 filter was a bandpass filter from 0.02 to 0.25 cycles/pixels, with Gaussian smoothed edges

152 (smoothing SD=0.005 cycles/pixel). After multiplying by these filters, we then replaced the 153 image's phase with random values uniformly sampled between -pi to +pi (to randomize the 
154 spatial phase of oriented elements in the image) and transformed back into the spatial domain

155 (using ifft2.m). Next, we cropped the image back to its original size of [224 x 224], multiplied

156 again by the smoothed circular mask, and converted the image into a 3-channel RGB format.

157 Finally, the luminance in each color channel was normalized to have a mean equal to the mean

158 of that color channel in the training ImageNet images and a standard deviation of 12 units. All

159 image processing for the evaluation image sets was done using Matlab R2018b (MathWorks,

160 Natick MA).

161

162 Using the above procedures, we generated four evaluation image sets, each starting with a

163 different random set of ImageNet images. Images in each evaluation set had orientations that

164 varied between $0^{\circ}$ and $179^{\circ}$, in steps of $1^{\circ}$, resulting in 180 discrete orientation values.

165 Throughout this paper, we use the convention of $0^{\circ}$ for vertical and $90^{\circ}$ for horizontal

166 orientations, with positive rotations referring to the clockwise direction, and negative rotations

167 referring to the counter-clockwise direction. Each evaluation set included 48 examples of each

168 orientation, for a total of 8640 images per set.

170 Measuring image set statistics

171

172 To verify that the modified versions of the ImageNet images had the anisotropic orientation

173 statistics that we expected, we measured the orientation content of each training image using a

174 Gabor filter bank. The filter bank included filters at orientations from $0^{\circ}$ to $175^{\circ}$ in $5^{\circ}$ steps, at

175 spatial frequencies of $0.0200,0.0431,0.0928$, and 0.200 cycles per pixel (orientation bandwidth 
176 of filters was $19^{\circ}$ ). The filter bank was generated using the gabor.m function in Matlab R2018b

177 (MathWorks, Natick MA). Since all filtering was performed in the Fourier domain, we also used

178 a custom modified version of the gabor.m function which allowed us to directly generate a

179 frequency-domain representation of each filter (Jain \& Farrokhnia, 1991). Before filtering each

180 image, we converted it to grayscale, subtracted its background color so that the background

181 was equal to zero, and padded each image with zeros to a size of $1012 \times 1012$ pixels (this was

182 the size needed to accommodate the lowest frequency filter). Images were then converted into

183 the frequency domain for filtering (using fft2.m), and multiplied by the filter bank. Next, we

184 converted back to the spatial domain and un-padded the image back to its original size ( $224 \mathrm{x}$

185224 pixels). Finally, we took the magnitude of the filtered image, and averaged the magnitude

186 across all pixel positions to obtain a single value for each filter orientation and spatial

187 frequency. Next, for each image, within each spatial frequency, we converted the orientation

188 magnitude values into an estimated probability distribution by dividing by the sum of the

189 magnitude across all orientations. Since this was done for all orientations of one spatial

190 frequency at a time, this correct for differences in power across spatial frequency and facilitates

191 combining results across spatial frequency. Results were similar within each spatial frequency

192 individually; we averaged over spatial frequency to produce the final plots (Figure 6B). This

193 analysis was done on the training set images only, which included 1300 images in each of 1000

194 categories, for a total of $\sim 1.3$ million images.

195

196 Network training and evaluation 
198 We trained VGG-16 networks (Simonyan \& Zisserman, 2014) on three different modified

199 versions of the ImageNet dataset (see Training stimuli for details). For each of the three image

200 sets, we initialized and trained four VGG-16 networks (replicates), giving a total of 12 models.

201 All models were trained using Tensorflow 1.12.0 (Abadi et al., 2016), using the TF-slim model

202 library (Silberman \& Guadarrama, 2016) and Python 3.6 (Python Software Foundation,

203 Wilmington DE). All models were trained using the RMSProp algorithm with momentum of 0.80

204 and decay of 0.90 . The learning rate was 0.005 with an exponential decay factor of 0.94 , and

205 the weight decay parameter was 0.0005 . Networks were trained until performance on the

206 validation set (categorization accuracy and top-5 recall) began to plateau, which generally

207 occurred after around 350K-400K steps. The validation images used to evaluate performance

208 were always rotated in an identical manner to the training set images. Training was performed

209 on an NVIDIA Quadro P6000 GPU (NVIDIA, Santa Clara CA). All evaluation was performed using

210 the first checkpoint saved after reaching 400K steps. As noted above, we did not perform data

211 augmentation steps during image pre-processing for training. Removing these procedures may

212 have contributed to the relatively low final classification performance that we observed (top-5

213 recall accuracy $\sim 60 \%)$.

215 To measure activations from each trained network, we split the evaluation image sets

216 (consisting of 8640 images each) into 96 batches of 90 each. We then passed each batch

217 through each trained network and measured the resulting activations of each unit as the output

218 of the activation function (a rectified linear operation). We saved the activations for each unit in

219 each layer for all images, which were then submitted to further analysis. We performed this 
220 evaluation procedure on a total of 17 networks: the 12 models trained on modified ImageNet

221 images, a pre-trained VGG-16 network from the TF-slim model library (Silberman \&

222 Guadarrama, 2016), and four randomly initialized VGG-16 models that served as a control. All

223 subsequent analyses were performed using Python 3.6 (Python Software Foundation,

224 Wilmington DE).

225

226 Computing Fisher information (FI)

228 To measure the ability of each network layer to discriminate small changes in orientation, we

229 estimated Fisher information (FI) as a function of orientation. To estimate FI for each network

230 layer, we first computed FI for each unit in that layer, then combined information across units.

231 Fl for each unit was computed based on the slope and variance of that unit's activation at each

232 point in orientation space, according to the following relation:

233

$$
F I_{i}(\theta)=\frac{\left(\frac{\partial f_{i}(\theta)}{d \theta}\right)^{2}}{v_{i}(\theta)}
$$

235 Where $f_{i}(\theta)$ is the unit's measured orientation tuning curve, and $v_{i}(\theta)$ is the variance of the 236 unit's responses to the specified orientation. We estimated the slope of the unit's tuning curve

237 at $\theta$ based on the difference in its mean response $\left(\mu_{i}\right)$ to sets of images that were $\Delta=4^{\circ}$ apart

238 (using different values of $\Delta$ did not substantially change the results).

239

$$
\left(\frac{\partial f_{i}(\theta)}{d \theta}\right) \cong \frac{\mu_{i}\left(\theta_{1}\right)-\mu_{i}\left(\theta_{2}\right)}{\Delta}
$$


241 Where

$$
\theta_{1}=\theta-\frac{\Delta}{2}
$$

$$
\theta_{2}=\theta+\frac{\Delta}{2}
$$

246 We presented an equal number of images (48) at each orientation, so the pooled variance was

247 calculated as:

$$
v_{i}(\theta)=\frac{v_{i}\left(\theta_{1}\right)+v_{i}\left(\theta_{2}\right)}{2}
$$

250 Finally, we summed this measure across units of each layer to obtain a population level

251 estimate of Fl. Note that this measure does not account for the covariance among units, see

252 Multivariate analyses for complementary approaches.

$$
F I_{\text {pop }}(\theta)=\sum_{i=0}^{\text {nUnits }} F I_{i}(\theta)
$$

255 Where $n U n i t s$ is the number of units in the layer. We computed $F I_{p o p}(\theta)$ for theta values

256 between $0^{\circ}$ and $179^{\circ}$, in steps of $1^{\circ}$. When plotting $\mathrm{FI}$, to aid comparison of this measure

257 across layers with different numbers of units, we divided $F I_{p o p}$ by the total number of units in

258 the layer, to capture the average FI per unit. We note that this analysis was performed across

259 all units at each layer, not excluding any units whose spatial receptive field was outside the

260 circular stimulus region. These non-responsive units contributed zero to the Fisher information 
261 sum at early layers. We note also that due to the different numbers of units per layer, the

262 absolute values of FI are not directly comparable across layers.

263

\section{Multivariate analyses}

In addition to calculating the sum of univariate Fisher information across all individual units at

267 each network layer (see previous section, Computing Fisher information) we were also

268 interested in evaluating the information content of multivariate patterns of activation across

269 entire layers of the network. To this end, we computed a multivariate version of Fisher

270 information, using the following expression (Abbott \& Dayan, 1999):

271

$$
F I_{\text {mult }}(\theta)=f^{\prime}(\theta)^{T} Q(\theta) f^{\prime}(\theta)+\frac{1}{2} \operatorname{Tr}\left[Q^{\prime}(\theta) Q^{-1}(\theta) Q^{\prime}(\theta) Q^{-1}(\theta)\right]
$$

272 Where $Q(\theta)$ is the pooled covariance matrix, computed as:

$$
Q(\theta)=\frac{Q\left(\theta_{1}\right)+Q\left(\theta_{2}\right)}{2}
$$

274 And $Q^{\prime}(\theta)$ was the estimated derivative of the covariance matrix, obtained as:

275

$$
\left(\frac{\partial Q(\theta)}{d \theta}\right) \cong \frac{Q\left(\theta_{1}\right)-Q\left(\theta_{2}\right)}{\Delta}
$$

276

277 Where $\theta_{1}$ and $\theta_{2}$ are as defined in the previous section. $\operatorname{Tr}$ denotes the trace operation, and $278 Q^{-1}(\theta)$ is the inverse of the covariance matrix. Values of $\theta$ and $f^{\prime}(\theta)$ were as defined in the 279 previous section. 
281 To make computing the covariance matrix computationally feasible, we first performed

282 principal components analysis on the activation matrix from each network layer (for each image

283 set, a matrix of size [8640 $x$ nUnits]), implemented using Scikit-learn in Python 3.6 (Python

284 Software Foundation, Wilmington DE). We then computed the above expression for Fisher

285 information using the scores for the top $\mathrm{N}$ principal components (PCs), for values of $\mathrm{N}$ ranging

286 from 2 to 47 (there were 48 images per orientation, so covariance matrix estimates became

287 unstable when using more features). Values were similar for different values of $\mathrm{N}$ (several

288 examples of varying $\mathrm{N}$ shown in Figure 4B).

290 In addition to computing multivariate Fisher information in this reduced-dimensionality space,

291 we used the principal components to estimate the dimensionality of the orientation

292 representations at each layer (Figure 5C). The purpose of this was to facilitate qualitative

293 comparisons of the representational structure across layers. To isolate the dimensions that

294 were related to orientation, and not to variability across images having the same orientation,

295 we first averaged across images of the same orientation (e.g. collapsing over any variability not

296 related to orientation). This resulted in a matrix of size [180 $\times \mathrm{N}$ ], where $\mathrm{N}$ is the total number

297 of units in the layer. Next, we performed PCA on this averaged matrix, and calculated the

298 cumulative variance explained by each principal component. Our estimate of dimensionality

299 was the PC number after which additional components contributed less than $5 \%$ additional

300 variance. This threshold is somewhat arbitrary but captures the approximate point at which an

301 "elbow" appears in a plot of cumulative variance versus PC number (Figure 5C). We note that 
302 this estimate of dimensionality is not exact and is not cross-validated, but should provide a

303 reasonable index of how dimensionality changes across model layers.

304

305 Finally, we computed an additional measure of multivariate orientation separability in the

306 reduced-dimensionality PC space. The rationale for this was to provide a complementary

307 measure to the Fisher information and ensure that the results we obtained with Fl were not

308 specific to that method. For this measure, as with $\mathrm{Fl}$, we computed a statistic for each point $\theta$ in

309 orientation space, using the responses to two orientations $\Delta=4^{\circ}$ apart (as before, changing $\Delta$

310 did not substantially change the results). This resulted in two "clouds" of points in N-

311 dimensional PC space, corresponding to the two orientations (see Figure S2). We first

312 exhaustively computed the Euclidean distances between all pairs of points in different clouds

$313\left(48^{\wedge} 2=2304\right.$ total distances $)$. Next, we computed a t-statistic for these distances: the mean of

314 all distance values divided by the standard deviation of all distance values. This measure

315 reflects the reliability of the separation between point clouds corresponding to different

316 orientations. We computed this measure for several different values of $\mathrm{N}$.

318 Fisher information bias (FIB)

320 To quantify the amount of bias (non-uniformity) in Fisher information at each layer of the

321 network, we computed a measure which we refer to as the Fisher information bias (FIB). For

322 the pre-trained model and the networks trained on upright images, we expected the network

323 to over-represent cardinal orientations, showing peaks in FI around vertical and horizontal. 
324 However, the models trained on rotated images were expected to show peaks rotated by a

325 specified amount relative to the cardinal orientations. To account for these different types of

326 bias, we computed three versions of the FIB: one that measures the height of peaks in FI

327 around the cardinal orientations (FIB-0), one that measures the height of peaks in FI that are

$32822.5^{\circ}$ counter-clockwise of the cardinals (FIB-22), and one that measures the height of peaks in

$329 \mathrm{Fl}$ that are $45^{\circ}$ counter-clockwise of the cardinals (FIB-45), relative to a baseline. The equation

330 for each FIB measure is as follows:

$$
F I B=\frac{F I_{\text {peaks }}-F I_{\text {baseline }}}{F I_{\text {peaks }}+F I_{\text {baseline }}}
$$

335 Where $F I_{\text {peaks }}$ is the sum of the $\mathrm{Fl}$ values in a range $\pm 10^{\circ}$ around the orientations of interest

$336\left(0^{\circ}\right.$ and $90^{\circ}$ for FIB- $-6,67.5^{\circ}$ and $157.5^{\circ}$ for FIB-22, and $45^{\circ}$ and $135^{\circ}$ for FIB-45), and $F I_{\text {baseline }}$ is

337 the sum of the $\mathrm{FI}$ values in a range $\pm 10^{\circ}$ around the orientation chosen as a baseline $\left(22.5^{\circ}\right.$ and

$\left.338112.5^{\circ}\right)$. Since $\mathrm{FI}$ is necessarily positive, each of these FIB measures can take a value between +1

339 and -1 , with positive values indicating more information near the orientations of interest

340 relative to the baseline (peaks in $\mathrm{FI}$ ), and negative values indicating less information near the

341 orientations of interest relative to baseline (dips in FI). An analogous method was used to

342 compute the bias in multivariate FI (Figure 4) as well as the multivariate t-statistic (Figure S2;

343 see previous section, Multivariate analyses). 
345 To test whether FIB differed significantly between trained models and the randomly initialized

346 (not trained) models, we performed non-parametric $t$-tests between FIB values corresponding

347 to each training set and the random models. Specifically, we tested the hypothesis that the

348 primary form of bias measured in models corresponding to each training set (e.g. FIB-0 for the

349 models trained on upright images, FIB-22 for the models trained on $22.5^{\circ}$ rotated images, FIB-

35045 for the models trained on $45^{\circ}$ rotated images) was significantly higher for the models trained

351 on that image set than for the random (not trained) models. Since we generated four replicate

352 models for each training image set, and evaluated each model on four evaluation image sets,

353 there were 16 total FIB values at each layer corresponding to each training set. To compare the

354 FIB values corresponding to each training set against the random models, we first calculated

355 the "real" difference in FIB between the groups, based on comparing the mean of the 16 values

356 for the trained models versus the mean of the 16 values for the random models. Next, we

357 concatenated the values for the trained and random models (32 values total) and randomly

358 shuffled the group labels across all values 10,000 times. For each of these 10,000 shuffles, we

359 then computed the difference between groups based on the shuffled labels ("shuffled"

360 differences). The final p-value was generated by calculating the number of iterations on which

361 the shuffled difference exceeded the real difference and dividing by the number of total

362 iterations. The $p$-values were FDR corrected across model layers at $q=0.01$ using SciPy

363 (Benjamini \& Yekutieli, 2001). The same procedure was used to test for differences in FIB-0

364 between the pre-trained model and the control model (note that there was only one replicate

365 for the pre-trained model, so this test included only 4 data points per condition). 


\section{Single-unit tuning analysis}

369 To measure the extent to which training set statistics impacted the orientation tuning of

370 individual units in each network, we measured tuning functions based on each unit's responses

371 to the evaluation image set, and we performed curve fitting to quantify tuning properties. First,

372 we measured an orientation tuning function for each unit at each layer of the model by

373 averaging its responses to all evaluation set images that had the same orientation (in each

374 image set, there were 48 images at each of 180 orientations). Any units that had a constant

375 response across all images or a zero response to all images were removed at this stage (this

376 included mainly units whose spatial selectivity was outside the range stimulated by the circular

377 image aperture, around 35\% of units per layer at the earliest layers). We computed and saved

378 an orientation tuning curve for each unit in response to each of the four evaluation image sets.

379 We then averaged over these four evaluation sets before fitting.

381 To characterize the tuning curves, we fit each with a circular Gaussian (Von Mises) function, 382 having the basic form:

$$
v(\theta)=e^{(k * \cos (\theta-u)-1)}
$$

386 Where $u$ is a parameter that describes the center of the unit's tuning function, and $k$ is a

387 concentration parameter that is inversely related to the width of the tuning function. In this

388 formulation, the $k$ parameter modifies both the height and the width of the tuning function. To 
make it possible to modify the curve's height and width independently, we normalized the Von Mises function to have a height of 1 and a baseline of 0 , and then added parameters for the

391 amplitude and baseline, as follows:

$$
f(\theta)=b+a * v_{n}(\theta)
$$

395 Where $v_{n}(\theta)$ denotes the Von Mises function after normalization. This resulted in a curve with

396 four total parameters: center $(u)$, concentration parameter $(k)$, amplitude, and baseline.

398 We fit a curve of this form to each unit's average tuning function using linear least-squares

399 regression, implemented with the optimization library in SciPy (version 1.1.0). To initialize the

400 fitting procedure, we used the argmax of the tuning function as an estimate of its mean, the

401 minimum value as an estimate of its baseline, and the range as an estimate of its amplitude.

402 The concentration parameter $k$ was always initialized at 1 . Values for the center were

403 constrained to lie within the range of [-0.0001, 180.0001], k was constrained to positive values

$404>10^{-15}$, and amplitude and baseline were allowed to vary freely. To prevent any bias in the

405 center estimates due to the edges of the allowed parameter range, we circularly shifted each

406 curve by a random amount before fitting.

407

408 After fitting was complete, we assessed the goodness of the fit using $\mathrm{R}^{2}$. To assess the

409 consistency of tuning across different versions of the evaluation image set, we used $\mathrm{R}^{2}$ to assess

410 the fit between the single best-fit Von Mises function (computed using the tuning function 
411 averaged over all evaluation image sets) and each individual tuning curve (there were four

412 individual tuning curves, each from one version of the evaluation image set). We then averaged

413 these four $R^{2}$ values to get a single value. We used a threshold of average $R^{2}>0.40$ to

414 determine which units were sufficiently well-fit by the Von Mises function, and retained the

415 parameters of those fits for further analysis.

417 Code Availability

418 All code is available on the author's Github page (link to be added upon publication).

\section{Results}

422 We measured the activation responses of several trained VGG-16 networks (Figure 1A)

423 (Simonyan \& Zisserman, 2014) presented with oriented images (Figure 1B) to evaluate whether

424 each network showed non-uniformities in its orientation representations across feature space.

425 First, we tested whether a pre-trained VGG-16 model (Silberman \& Guadarrama, 2016) exhibits

426 the classical oblique effect. Next, we evaluated whether this bias changed in a predictable way

427 when networks with the same architecture were trained on modified versions of the ImageNet

428 database (Figure 6A). 
A

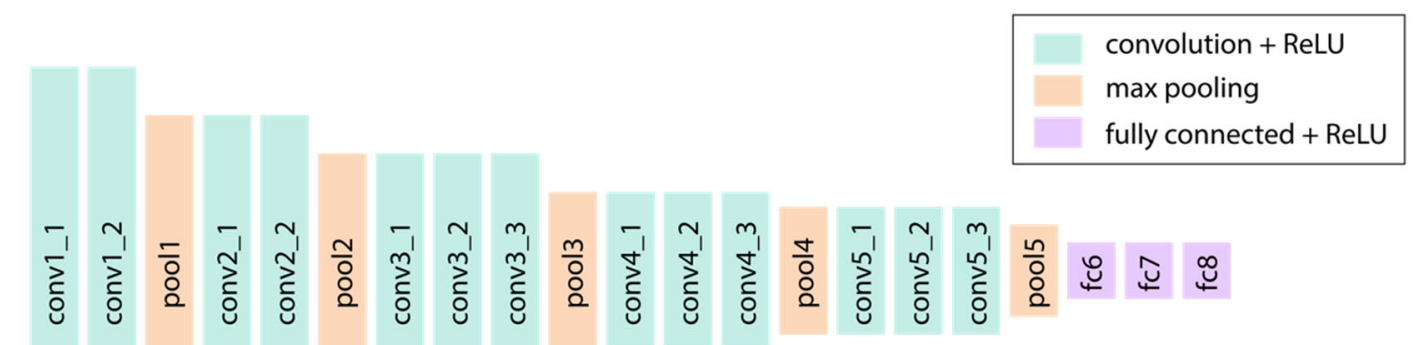

B
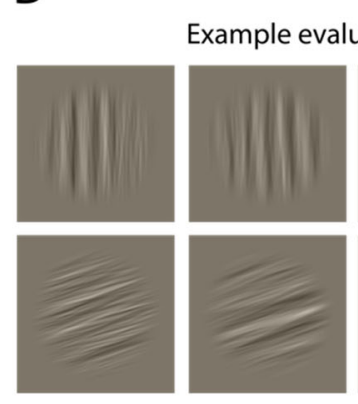

430

431

432

433

434

orientation range, preserving their broadband spatial frequency content. Orientations varied

436

between $0-179^{\circ}$, in steps of $1^{\circ}$ (see Methods, Evaluation stimuli). (C) Cartoon depiction of the

437 approximate relationship between an example single unit tuning function and the Fisher

438 information (FI) measured from that unit as a function of orientation. (D) Hypothetical

439 depiction of the relationship between the prior distribution $\mathrm{P}$ over orientation $\theta$, and the Fisher

440 information (FI) when mutual information is maximized (Ganguli \& Simoncelli, 2011; Wei \&

441 Stocker, 2015)

442

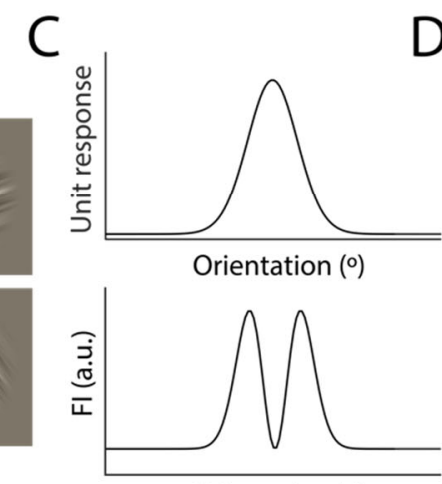

Orientation $\left({ }^{\circ}\right)$
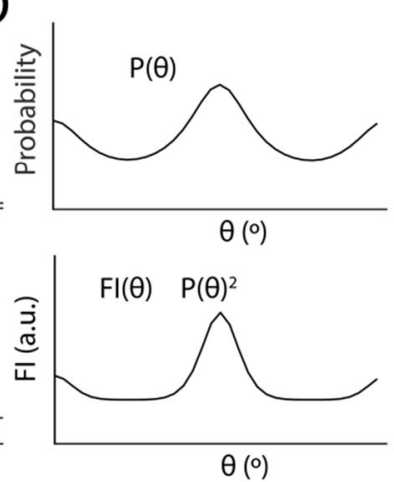

Figure 1. Evaluating orientation discriminability in a trained neural network model. (A)

Schematic of the VGG-16 network architecture, with layers arranged from shallowest (left) to deepest. (B) Examples of oriented images used to measure orientation representations in the pre-trained network. Images were generated by filtering ImageNet images within a narrow 
445 We first evaluated non-uniformity at the level of each pre-trained network layer by computing

446 the layer-wise Fisher information (FI), which reflects how well each layer's activations can

447 distinguish small changes in orientation (see Methods, Computing Fisher information). Briefly,

448 the contribution of each network unit to the layer-wise Fl is the squared slope of a unit's tuning

449 function at each orientation normalized by the variance of the response at that orientation.

450 Thus, the steep part of a unit's tuning function will carry more information because two similar

451 orientations will evoke different responses (Figure 1C). However, the flat parts of a unit's tuning

452 curve (i.e. at the peak or in the tails) will not carry very much information because the unit will

453 respond about the same to two similar orientations. We focused on the Fisher information

454 because it has been suggested to have a predictable relationship to the prior orientation

455 distribution. Specifically, when mutual information is maximized, Fisher information is

456 predicted to be proportional to the square of the prior distribution (Figure 1D; Ganguli \&

457 Simoncelli, 2011; Wei \& Stocker, 2015). This therefore provides a testable prediction of the

458 efficient coding account. To introduce the variability necessary for calculating Fisher

459 information, we used orientation bandpass-filtered natural images which create stimulus-level

460 variability in the images for a given orientation.

461

462 For a pre-trained VGG-16 model, plotting FI as a function of orientation reveals noticeable

463 deviations from uniformity, particularly at deep layers of the network (navy blue curves in

464 Figure 2A). While the first layer of the model (conv1_1), gives a relatively flat profile of FI with 
465 respect to orientation, by layer 7 (conv3_1), peaks in FI are apparent around the cardinal

466 orientations, $0^{\circ} / 180^{\circ}$ and $90^{\circ}$. At later layers of the model, the peaks in $\mathrm{Fl}$ are more

467 pronounced and begin to take on a characteristic double-peaked shape, where Fl is maximal

468 slightly $\left(\sim 5^{\circ}\right)$ to the left and right of the cardinal orientations, with a dip at the exact position of

469 the cardinal orientations (this shape is discussed in more detail in the next section after we

470 report statistics about the preferred orientation and width of single unit tuning functions). In

471 contrast, when the same analysis is done on a randomly initialized VGG-16 model (no training

472 performed), $\mathrm{Fl}$ is flat with respect to orientation at all layers, suggesting that a randomly

473 initialized model does not exhibit this same cardinal bias (gray curves in Figure 2A).

474

475

476

477

478

479 

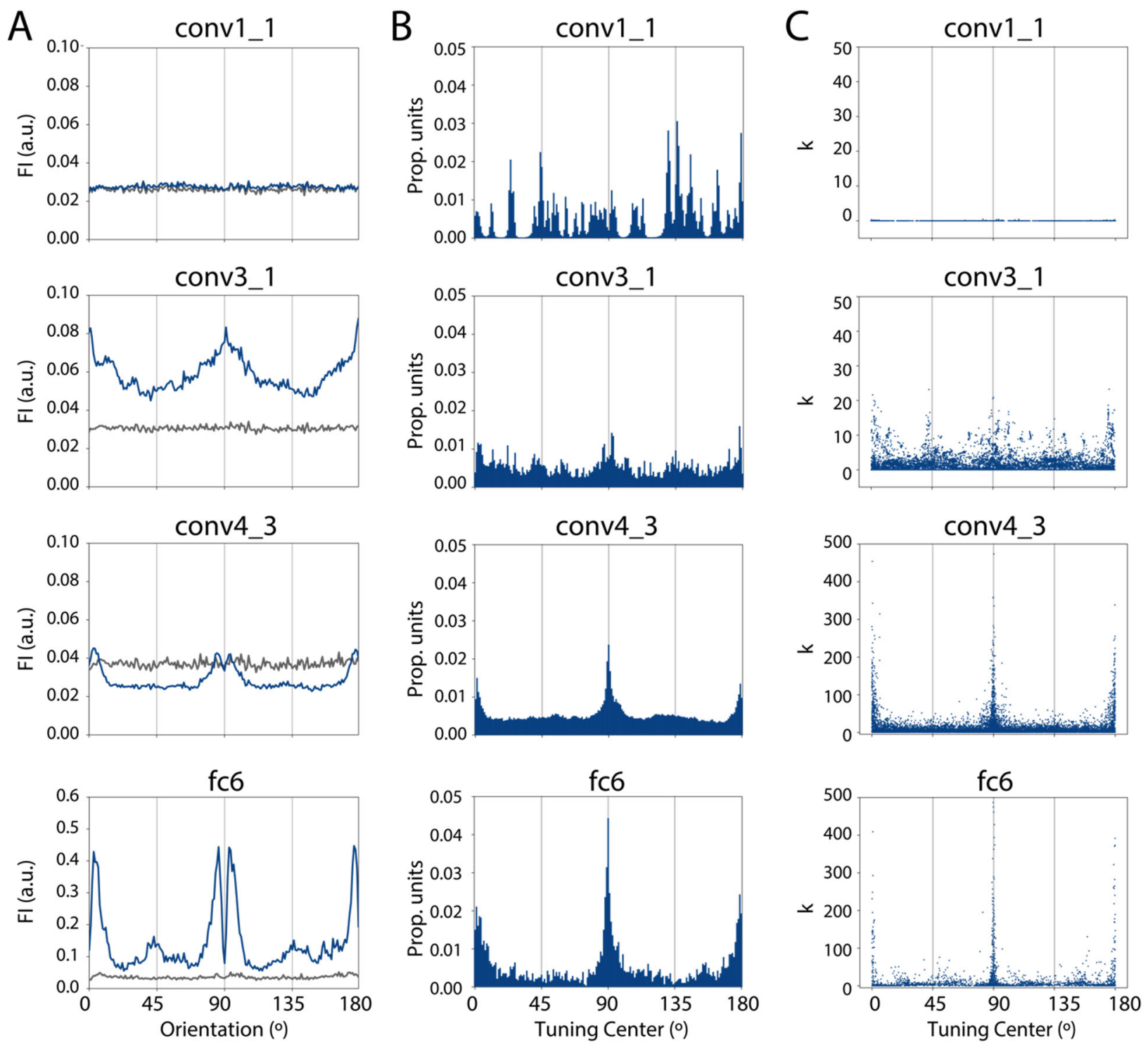

481 Figure 2. Pre-trained VGG-16 shows maximum orientation information just off of cardinal

482 orientations, and non-uniformity in the distribution of single unit tuning properties. (A) Fl is

483 plotted as a function of orientation for several example layers of the pre-trained model (navy

484 blue) and a randomly initialized model (gray). See Methods, Computing Fisher information for

485 details. (B) Distribution of the tuning centers of pre-trained network units that were well-fit by

486 a Von Mises function. See Figure S1 for the proportion of well-fit units per layer, and the

487 distribution of centers for the randomly initialized model. (C) Concentration parameter (k) 
versus center for individual units in the pre-trained model (data in the top three panels of $C$ have been down-sampled to a maximum of 10,000 points for visualization purposes).

491 To quantify this effect at each layer, we computed a metric which we term the Fisher

492 Information Bias (FIB), which captures the relative height of the peaks in FI compared to a

493 baseline (see Methods, Fisher information bias). We defined three versions of this metric, the

494 FIB-0, FIB-22, and FIB-45, which denote the height of peaks in FI around the cardinal

495 orientations, around orientations $22.5^{\circ}$ counter-clockwise of cardinals, and around orientations

$49645^{\circ}$ counter-clockwise of cardinals, respectively. For example, to get the FIB-0, we take the

497 mean $\mathrm{Fl}$ in $20^{\circ}$ bins around $0^{\circ}$ and $90^{\circ}$, subtract the mean $\mathrm{Fl}$ in in a baseline orientation range,

498 and divide by the sum of these two means. Because the pre-trained model showed peaks in FI

499 around cardinals only, we focus on the FIB-0 in this section; the FIB-22 and FIB-45 are discussed

500 in the following section (Training networks on rotated images). We found that for the pre-

501 trained model, the FIB-0 increased with depth in the network, showing values close to zero for

502 the first four layers, then showing positive values that increase continuously at each layer (navy

503 blue line in Figure 3). In contrast, we found less evidence for a cardinal bias in the randomly

504 initialized model, shown by smaller values of the FIB-0 at all layers (gray line in Figure 3). The

505 difference in FIB-0 between the pre-trained and randomly initialized models was significant

506 starting at the fifth layer (conv2_2), and at all layers deeper than conv2_2 (one-tailed non-

507 parametric t-test, FDR corrected q=0.01). However, there was a small increase in the FIB-0 at

508 the later layers of the randomly initialized model, reflecting a weak cardinal bias (at the deepest 
509 layer, the FIB-0 was still more than $5 x$ as large for the pre-trained model as for the random

510 model). We return to this issue for more consideration in the Discussion.

511

512

513

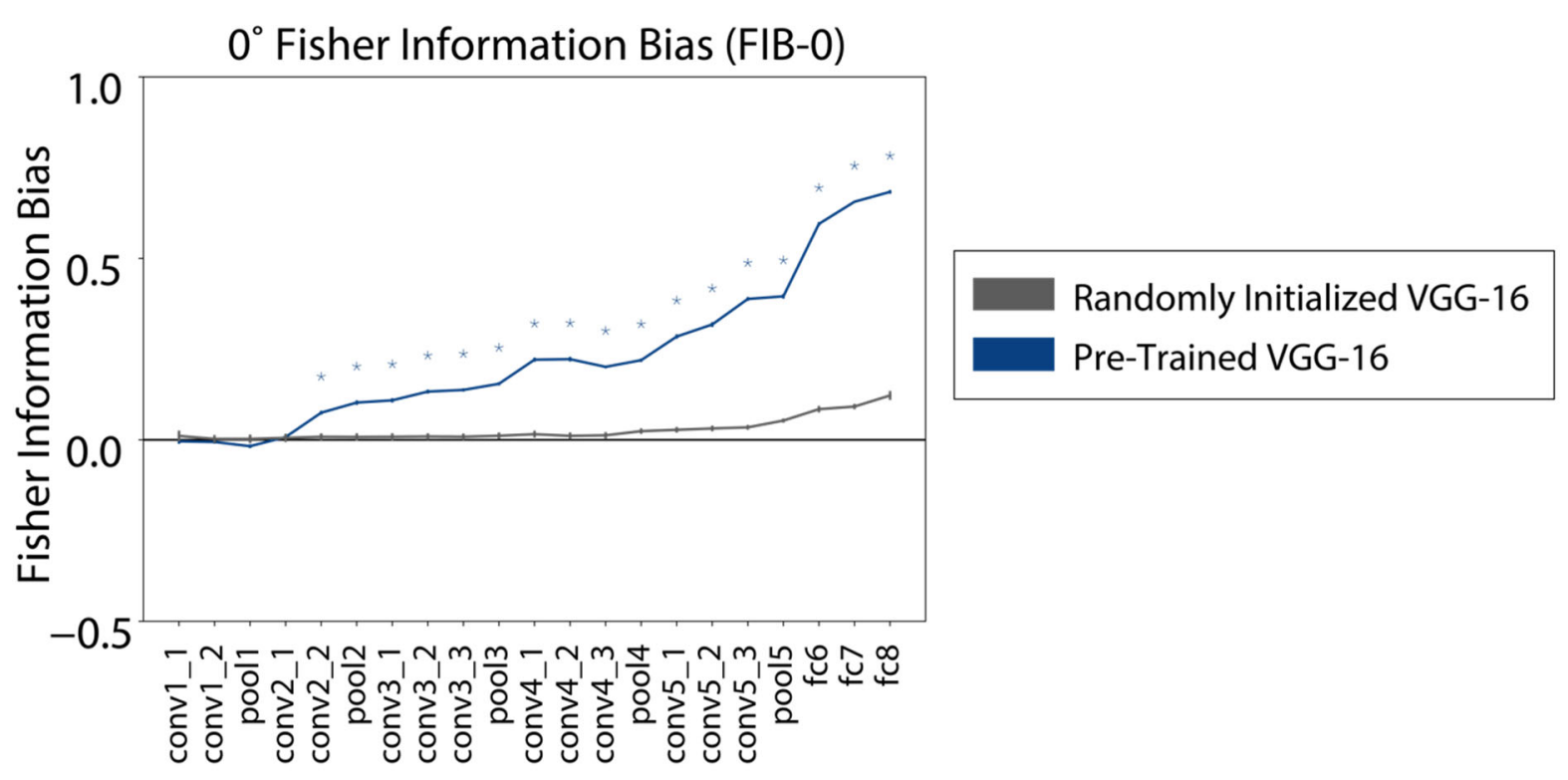

514

515 Figure 3. Cardinal bias in a pre-trained VGG-16 model increases with depth. FIB-0, a measure of

516 cardinal information bias (see Methods, Fisher information bias), plotted for a pre-trained

517 model (navy blue) and a randomly initialized control model (gray), with asterisks indicating

518 layers for which the pre-trained model had significantly higher FIB-0 than the random model

519 (one-tailed nonparametric t-test, FDR corrected $\mathrm{q}=0.01$ ). Error bars reflect standard deviation

520 across four evaluation image sets.

521 
522 Having demonstrated that a pre-trained CNN exhibits an advantage for discriminating cardinal

523 versus other orientations, we were next interested in whether this bias was linked to the

524 distribution of tuning properties across single units at each layer, as has been observed in the

525 brains of animals such as cats and macaques (Li et al., 2003; Shen et al., 2014; Vogels \& Orban,

526 1994). To investigate this, we computed the average orientation tuning profiles for individual

527 units in response to stimuli of all orientations and fit these profiles with Von Mises functions to

528 estimate their center and concentration parameter (or width, denoted $k$ ). Units that were not

529 well-fit by a Von Mises were not considered further (approximately $30 \%$ of all units, see

530 Methods, Single-unit tuning analysis and Figure S1. Figure 2B shows the distribution of fit

531 centers for all units in four example layers of the pre-trained model that were well-fit by a Von

532 Mises function. These distributions show peaks at random locations for the first layer of the

533 network, but exhibit narrow peaks around the cardinal orientations for the deeper conv4_3 and

534 fc6 layers. In contrast, the randomly initialized model did not show an over-representation of

535 cardinal-tuned units (Figure S1). In addition, plotting the concentration parameter for each unit

536 versus the center (Figure 2C) shows that for the deepest three layers shown, the most

537 narrowly-tuned units (high k) generally have centers close to the cardinal orientations.

538 Together, these findings indicate that middle and deep layers of the pre-trained network have a

539 large proportion of units tuned to cardinal orientations, and that many of these units are

540 narrowly tuned.

541

542 These findings may provide an explanation for the double-peaked shape of the FI curves for the

543 pre-trained model at deep layers (Figure 2A). Since Fl is related to the slope of a unit's tuning 
544 function, it is expected to take its maximum value on the flanks of a tuning curve, where slope

545 is highest, and take a value of zero at the tuning curve peak (Figure 1C). Thus, having a large

546 number of narrowly-tuned units with their peaks precisely at $0^{\circ}$ and $90^{\circ}$ could result in layer-

547 wise FI having local maxima at the orientations just off of the cardinals. We note also that these

548 findings indicate a divergence from the predicted shape of FI based on mutual information

549 maximization (Figure 1D); we return to this issue for further consideration in the Discussion.

551 In addition to the above analyses, we were also interested in determining whether biases were

552 present in activation patterns across all units in each layer. To achieve this, we computed the

553 multivariate Fisher information (see Methods, Multivariate analyses). Note that this is distinct

554 from the version of Fl described earlier in this section (Figure 2, Figure 3), which combines

555 information linearly across units but does not take advantage of the covariance structure of the

556 data. Before calculating multivariate $\mathrm{Fl}$, we first reduced the dimensionality of the data using

557 principal components analysis (PCA; see Methods, Multivariate analyses). As shown in Figure 4,

558 multivariate $\mathrm{Fl}$ revealed a similar pattern of results as the summed univariate $\mathrm{FI}$ discussed

559 previously. FI was generally flat across orientation space for the earliest layers, and peaks

560 around the cardinal orientations began to emerge at the middle layers (around conv3_1, for

561 this analysis). As with the univariate version of $\mathrm{Fl}$, multivariate $\mathrm{Fl}$ at the deeper layers of the

562 model exhibited a pronounced double-peaked shape with highest values a few degrees

563 clockwise and counter-clockwise of the cardinal orientations. Furthermore, when we utilized

564 another measure of orientation discriminability in PC space, based on the reliability of distances

565 between points corresponding to nearby orientations (Figure S2, see Methods, Multivariate 
566 analyses), we again found biases that were similar in form and emerged at middle layers of the

567 model. In contrast to FIB, the cardinal bias in this measure did not exhibit a continual increase

568 with depth in the model, however it was above zero for all middle and late layers. Together,

569 these results suggest that the presence of pattern-level orientation biases in VGG-16, and the

570 fact that these biases persist until the deepest model layers, is not dependent on the specific

571 information metric used.

572

573

574 

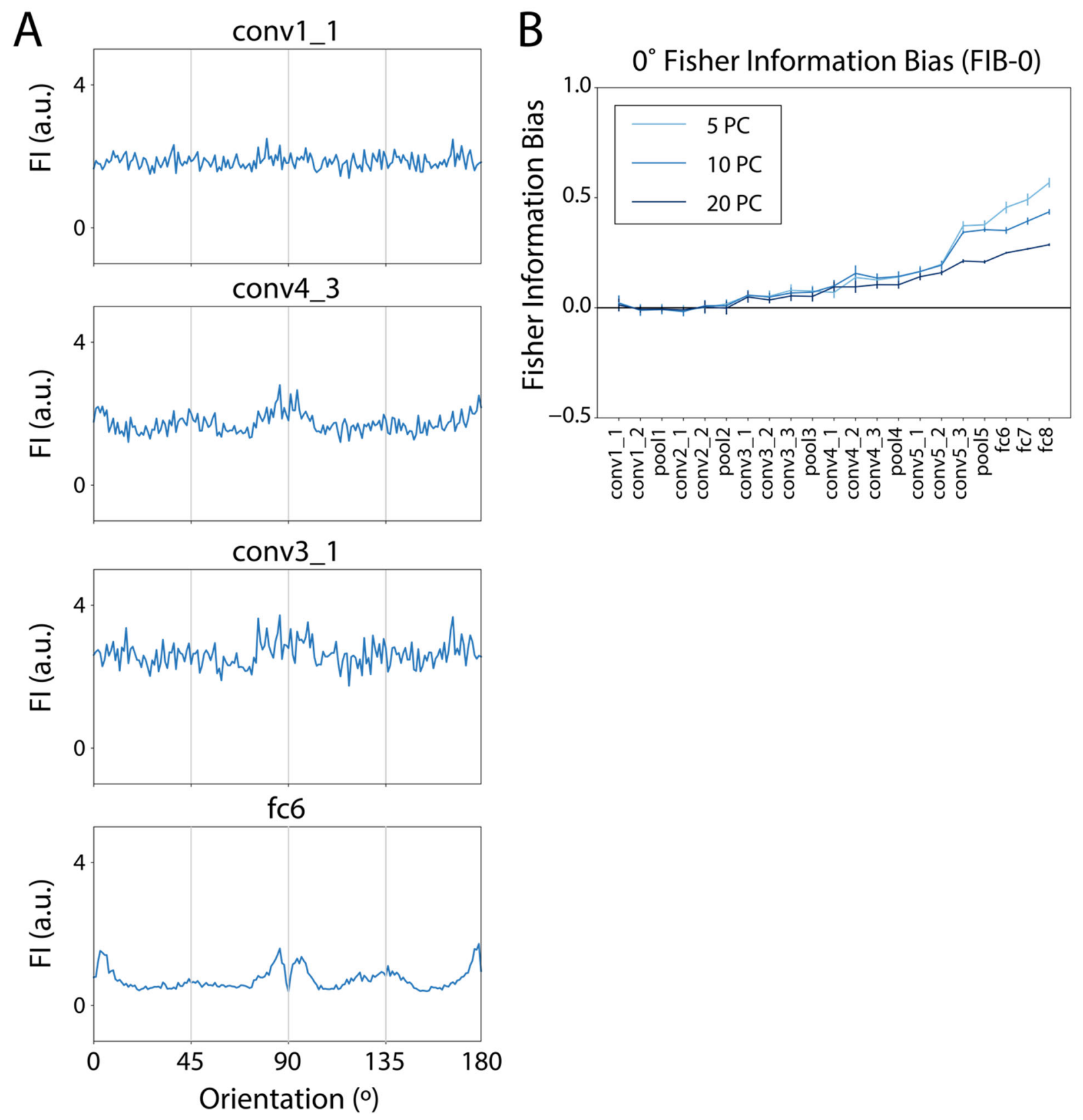

576 Figure 4. Multivariate FI shows similar pattern of results as summed univariate FI. (A)

577 Multivariate Fisher information, calculated after performing PCA, shown for four example

578 layers in the pretrained model. See methods (Multivariate analyses) for details. This figure

579 reflects the calculation with first 10 PCs retained; similar results were found with different

580 numbers of PCs retained. (B) Multivariate version of FIB-0 is plotted for each layer, comparing 
581 results with 5, 10 or 20 principal components retained. Error bars reflect \pm 1 standard deviation

582 of the measure across 4 evaluation image sets.

584 The above univariate and multivariate analyses suggest that the structure of orientation

585 representations differs at early, middle, and deep layers of VGG-16. To further explore this

586 difference and visualize the representations at each layer, we plotted the representations of

587 images in the space spanned by the first two principal components (Figure 5A). We also plotted

588 the orientation tuning profiles of the first four principal components of several example layers

589 (Figure 5B). This revealed that at the earliest layer of the model (conv1_1), the representation

590 of orientation appeared to be well-described by two principal components resembling a sine

591 and cosine function, resulting in an approximately circular representation in PC space. The third

592 and fourth principal components for this layer showed little response change as a function of

593 orientation. In contrast, the middle and deeper layers of the model exhibited more complex

594 response profiles. At conv3_1 and conv4_3, PCs 1 and 2 were similar to those of the first layer,

595 but the third and fourth principal components tended to be higher frequency or include sharp

596 points near the cardinal orientations. At a deep fully-connected layer ( $f(6)$, the tuning profiles

597 were no longer sinusoidal, and instead exhibited steep points and/or dips near the cardinal

598 axes. The steepest regions of these principal component tuning profiles appear to coincide with

599 the peaks we measured in FI (Figure 2A, Figure 4), suggesting these response profiles may

600 underly the strong biases we observed at deep layers.

601 
602 Additionally, these more complex response profiles may imply an increase in the dimensionality

603 of the space spanned by all distinct orientations. To estimate the dimensionality of this

604 subspace, we averaged over different images at each orientation, and performed PCA on the

605 resulting matrix (see Methods, Multivariate analyses). When we plot the cumulative percent

606 variance explained by each component in this representation (Figure 5C), we find distinct

607 patterns between early and late layers. While early layers exhibit a clear plateau where

608 components after 2 contribute little additional variance, later layers exhibit a smoother

609 function where more components are required to reach a plateau. Together, these

610 observations support the idea that the dimensionality of orientation representations increases

611 from shallow to deeper layers of VGG-16, with representations morphing from a veridical

612 representation of the circular feature space spanned by orientation, to a more complex and

613 biased format.

614 


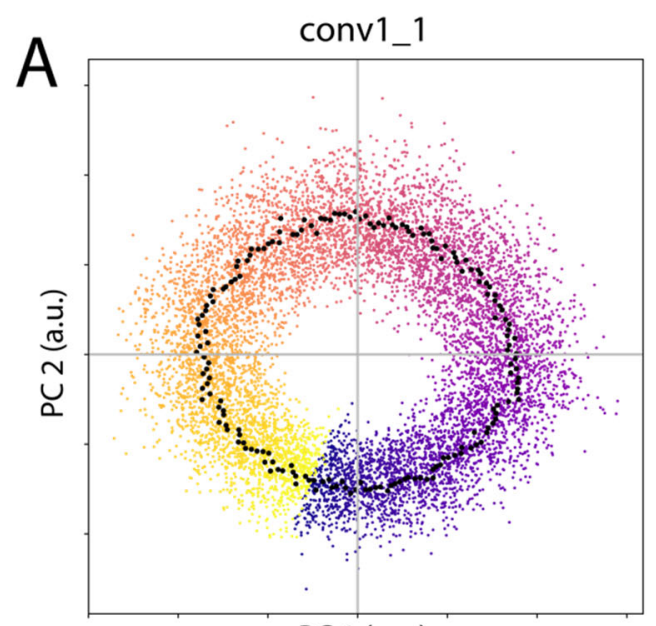

PC 1 (a.u.)

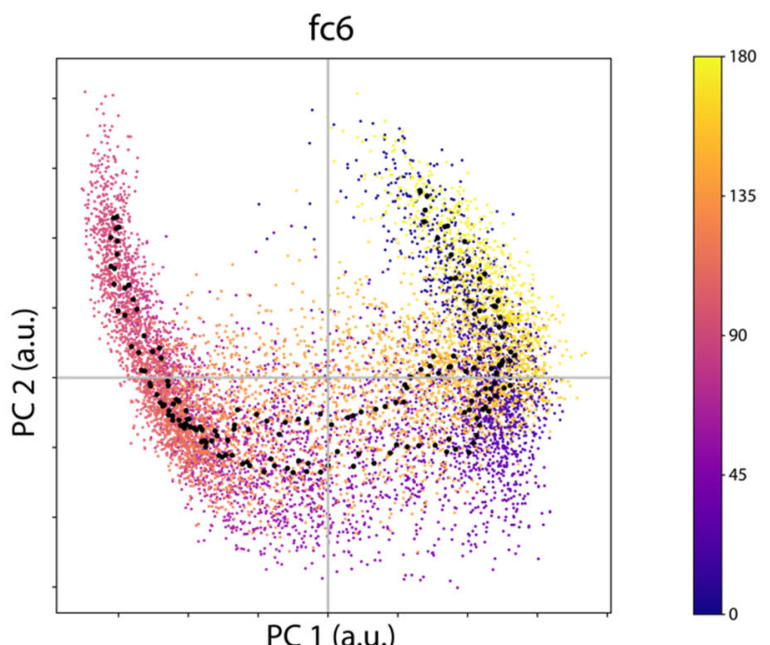

PC 1 (a.u.)

B
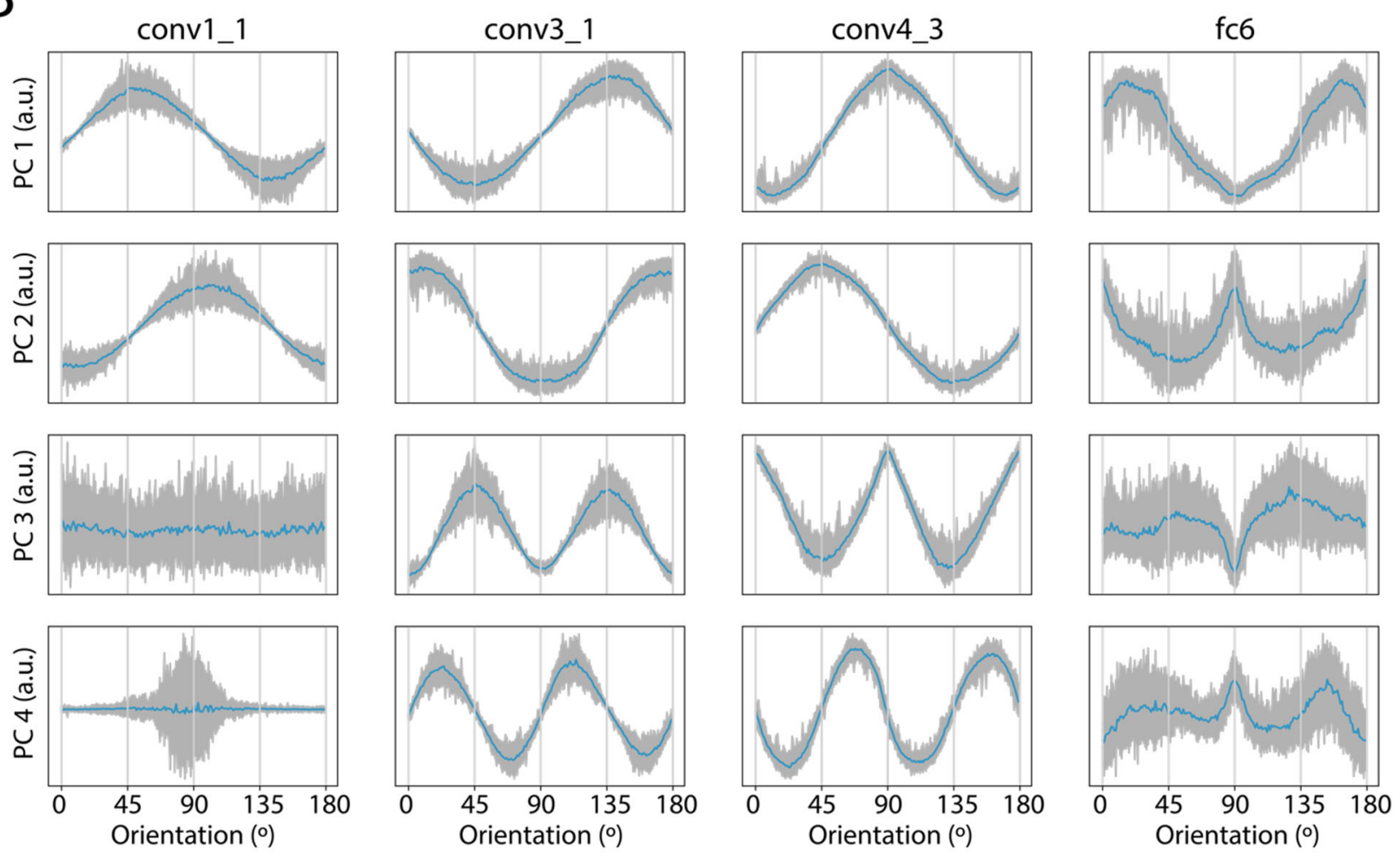

C
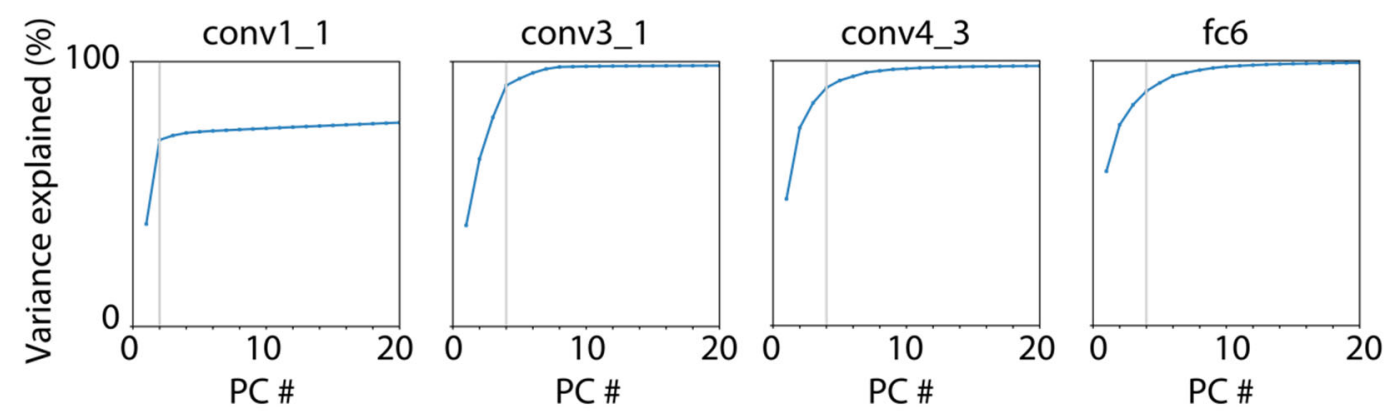
616 Figure 5. Principal component analysis reveals a graded change in the structure of orientation

617 representations across pre-trained model layers. (A) For two example layers (conv1_1 and fc6),

618 scatter plot of scores corresponding to the first two principal components of the layer's

619 representation (see Methods, Multivariate analyses). Colored points indicate individual images,

620 with color indicating stimulus orientation. Black points indicate the mean of the 48 points

621 corresponding to each orientation. (B). Scores for the first four principal components are

622 plotted as a function of orientation, for several example layers. Blue lines indicate the mean

623 value of that principal component score as a function of orientation, gray lines indicate

624 individual images. (C) Percent variance explained by each principal component of the data,

625 after averaging across trials of a common orientation. Vertical line indicates the number of

626 components after which additional components contribute $<5 \%$ additional variance (see

627 Methods, Multivariate analyses).

628

629 Training networks on rotated images

630

631 Having demonstrated that a pre-trained VGG-16 network exhibits a much stronger cardinal

632 orientation bias compared to a randomly initialized network, we next tested whether training a

633 model on rotated images would result in rotated biases. This test is needed to demonstrate

634 that the frequently-observed cardinal bias is not the only possible orientation bias that can be

635 induced in a visual system through exposure to a set of images with non-uniform statistics. We

636 trained networks on three modified versions of the ImageNet dataset (Deng et al., 2009),

637 consisting of images that were rotated by either $0^{\circ}, 22.5^{\circ}$, or $45^{\circ}$ in a clockwise direction 
638 relative to the upright position (Figure 6A). Separately, we also verified that the image statistics

639 of each of the modified sets exhibited the expected distribution, such that vertical and

640 horizontal orientations were most common in the upright training set, orientations $22.5^{\circ}$

641 counter-clockwise of cardinals were most common in the $-22.5^{\circ}$ rotated set, and orientations

$64245^{\circ}$ counter-clockwise of cardinals were most common in the $-45^{\circ}$ rotated set (Figure $6 \mathrm{~B}$ ).

643 Given the hypothesized relationship between $\mathrm{Fl}$ and the prior probability distribution (Figure

644 1D), we predicted that the FI curves for each of these models would have peaks closely aligned

645 with the peaks of its respective prior distribution.

646

647

648 
A

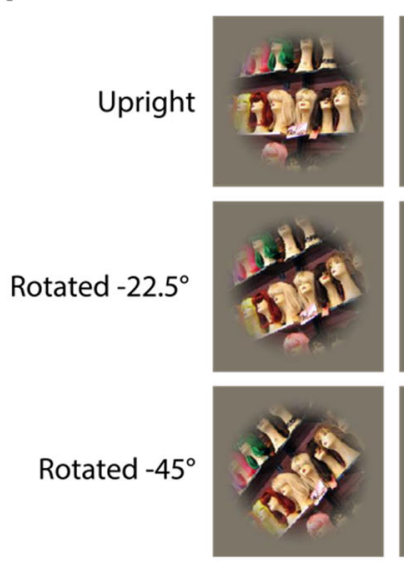

B ImageNet (upright)

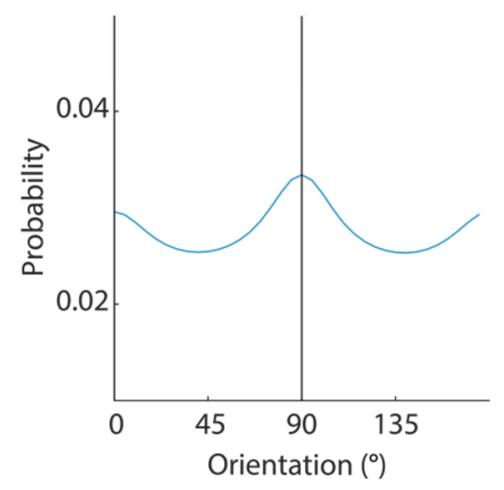

Example training images
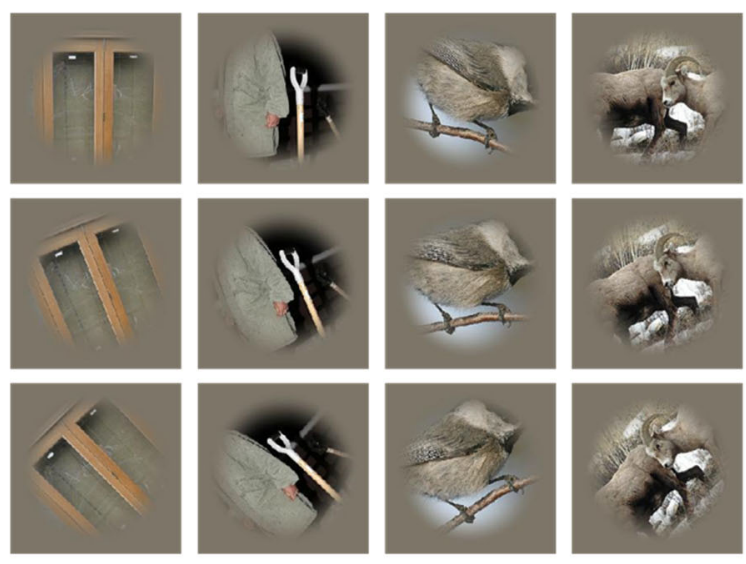

ImageNet rotated $-22.5^{\circ}$

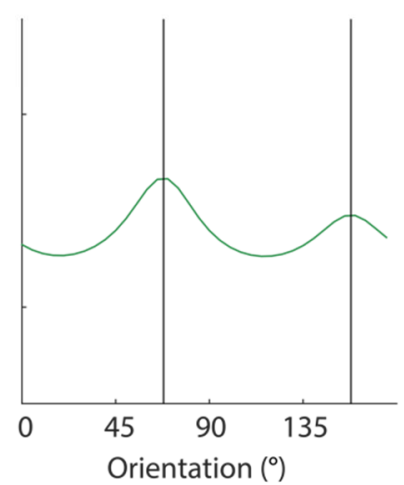

ImageNet rotated $-45^{\circ}$

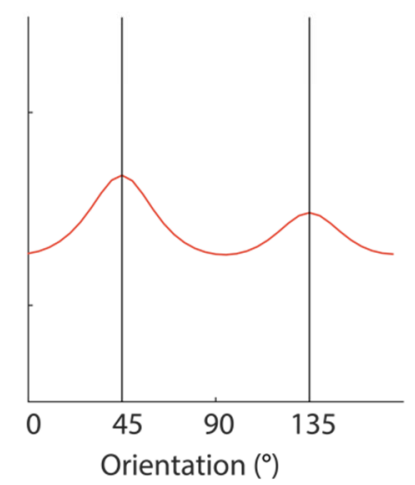

Figure 6. Rotated images used to train VGG-16 networks. (A) Separate networks were trained

651 on either upright or rotated versions of the ImageNet image set, with a smoothed circular mask

652 applied to remove vertical and horizontal image edges. (B) Orientation content from images in

653 each of the training sets in (A) was measured using a Gabor filter bank (see Methods,

654 Measuring image set statistics).

655

656 Our results indicate that training on rotated images shifted the orientation bias by a predictable

657 amount. Fl for the models that were trained on upright images shows a relatively similar shape

658 to the pre-trained model, with peaks appearing at a few degrees to the left and right of the

659 cardinal orientations (Figure 7A). This demonstrates that though our training procedure and 
660 image set were not identical to those used for the pre-trained model, they resulted in the

661 formation of similar orientation biases. In contrast, the models trained on rotated images each

662 showed a Fl curve that was similar in shape but shifted relative to the curve from the model

663 trained on upright images, such that the peaks in Fl were always near the orientations that

664 were most common in the training set images (Figure 7D,7G).

665

666 The distribution of single-unit tuning properties also shifted with training set statistics. In the

667 upright-trained model, the highest proportion of units had their tuning near the cardinals, while

668 the networks trained on $22.5^{\circ}$ and $45^{\circ}$ rotated images had more units with tuning at either

$66922.5^{\circ}$ or $45^{\circ}$ counter-clockwise relative to the cardinal orientations, respectively (Figure

$6707 \mathrm{~B}, 7 \mathrm{E}, 7 \mathrm{H})$. Additionally, for all models, the most narrowly-tuned units tended to be those that

671 were tuned to the orientations most common in the training set (Figure $7 \mathrm{C}, 7 \mathrm{~F}, 7 \mathrm{I})$. As described

672 above, the high number of narrowly-tuned units with their centers close to these most common

673 orientations may underly the double-peaked shape seen in FI. 
A

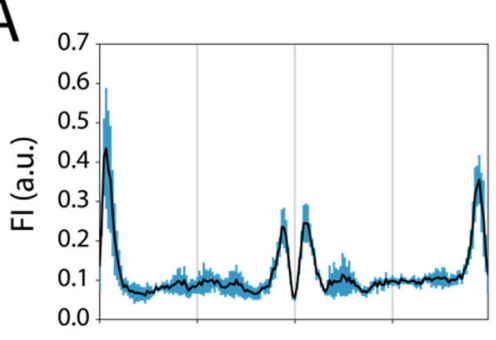

D

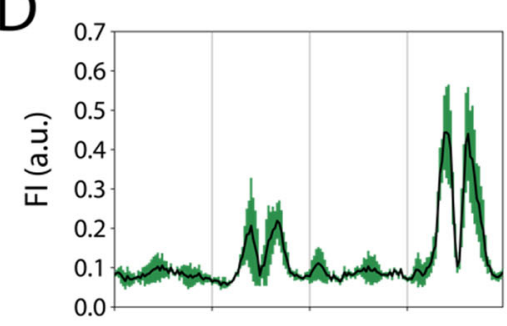

G

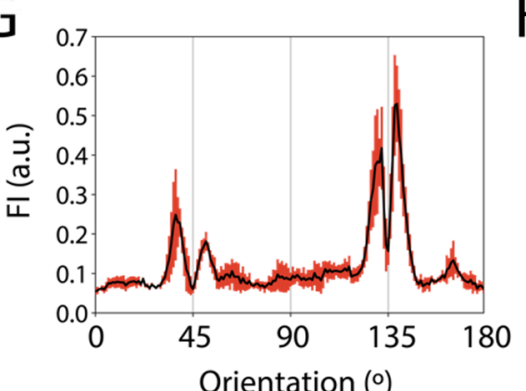

B

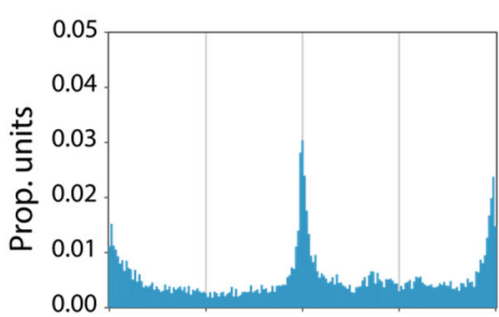

$\mathrm{E}$

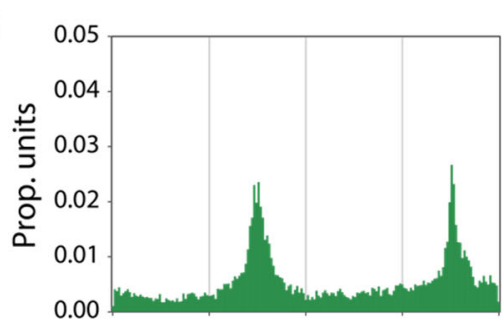

$\mathrm{H}$

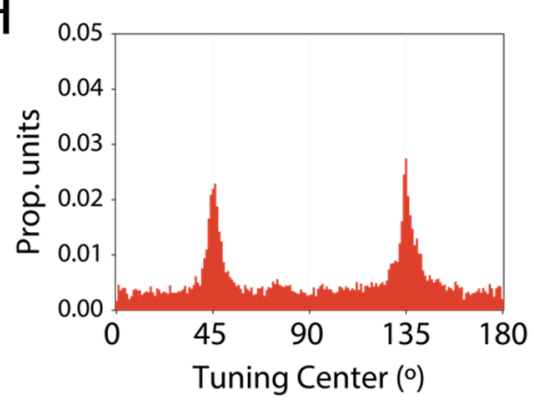

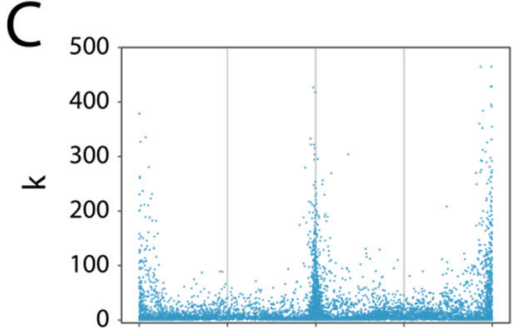

$\mathrm{F}$
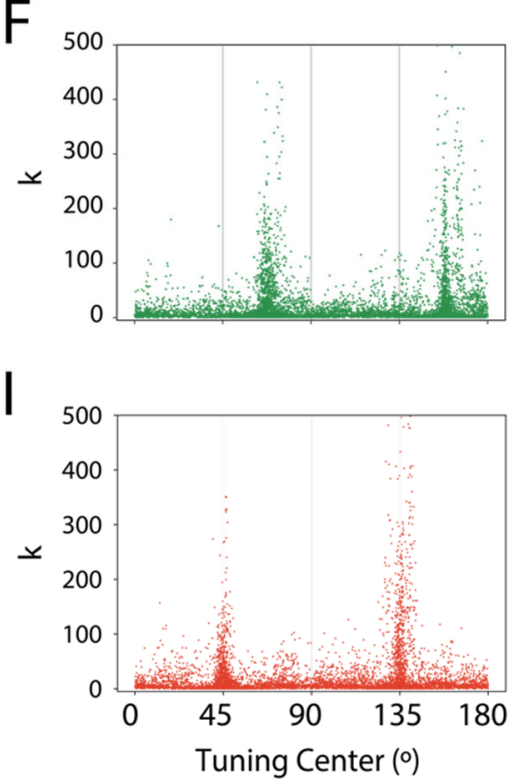

Figure 7. When networks are trained on rotated images, both population-level information and

676 single unit tuning distributions reflect modified training set statistics. (A-C) show data from one

677 example layer (fc6) of four separately initialized networks trained on upright images, (D-F) show

678 data for fc6 of networks trained on images rotated $22.5^{\circ}$ counter-clockwise of upright, (G-I)

679 show data for fc6 of networks trained on images rotated $45^{\circ}$ counter-clockwise of upright. For

680 each group of networks, panels $(\mathbf{A}, \mathbf{D}, \mathbf{G})$ show Fl plotted as a function of orientation, with error

681 bars reflecting standard deviation across four networks with the same training image set

$682(\mathbf{B}, \mathbf{E}, \mathbf{H})$ show distribution of fc6 unit tuning centers, combining data across networks $(\mathbf{C}, \mathbf{F}, \mathbf{I})$

683 show concentration parameter $(k)$ versus center for individual units. 
685 Calculating the FIB for each of these models further demonstrated how these effects emerged

686 across the processing hierarchy. Like the pre-trained model, the models trained on upright

687 images showed high values of the FIB-0 at middle and deep layers: models showed significantly

688 higher FIB-0 than the randomly initialized models for pool1, conv3_1, and all layers deeper than

689 conv3_1 (one-tailed nonparametric t-test, FDR corrected q=0.01) (Figure 8A). In contrast, the

690 models trained on images rotated by $22.5^{\circ}$ and $45^{\circ}$ showed higher values for the FIB-22 and

691 FIB-45, respectively (Figure 8B,8C). In models trained on images rotated by $22.5^{\circ}$, the FIB-22

692 significantly exceeded that of the random models at pool2 and all layers deeper than pool2,

693 with the exception of conv3_3 (one-tailed nonparametric t-test, FDR corrected q=0.01). For the

694 models trained on $45^{\circ}$ rotated images, the FIB-45 significantly exceeded that of the random

695 models for conv3_1 and all layers deeper than conv3_1 (one-tailed nonparametric t-test, FDR

696 corrected $\mathrm{q}=0.01)$.

697 

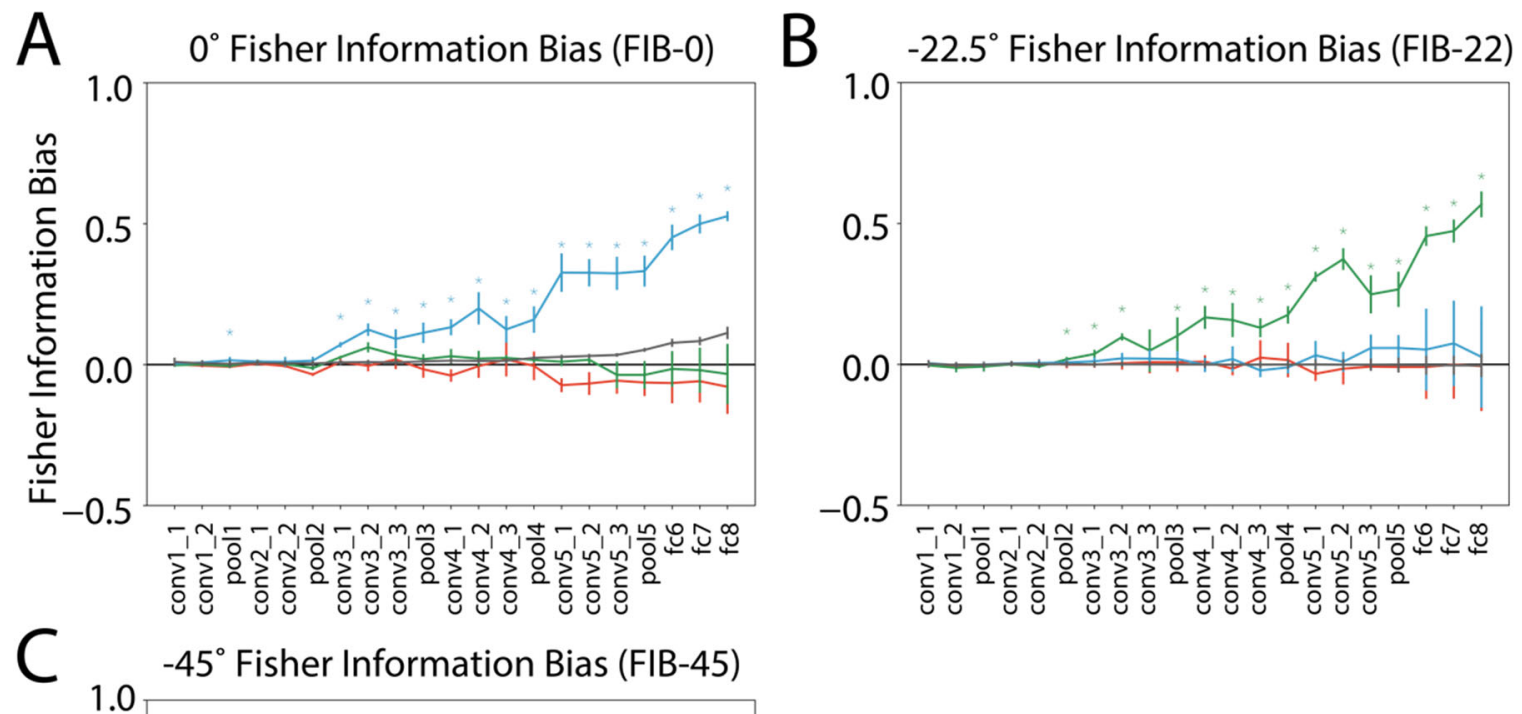

Figure 8. Networks shows biases in orientation discriminability that are consistent with training set statistics. FIB-0, FIB-22, and FIB-45 represent the relative value of FI at cardinal orientations, $22.5^{\circ}$ counter-clockwise of cardinals, and $45^{\circ}$ counter-clockwise of cardinals, respectively, relative to a baseline (see Methods, Fisher information bias). Panels show (A) FIB-0, (B) FIB-22, and (C) FIB-45 for models trained on each rotated version of ImageNet (colored), and randomly initialized models (gray). Colored asterisks indicate layers for which the models corresponding to that color had significantly higher FIB than the random models (one-tailed nonparametric ttest, FDR corrected $q=0.01$ ). Error bars represent the standard deviation of the FIB over four

707 initializations of each model and four evaluation image sets. 
Emergence of biases during training

711 Though we have demonstrated that biases were measurable in each fully trained model, this

712 leaves open the question of whether and how these biases relate to the model's performance

713 at its primary task (i.e. categorizing object images). One way to address this is by examining

714 how biases emerged over time during the training process. We hypothesized that if biases

715 contribute to the model's ability to learn the object classification task, then we should observe

716 strong biases early in training, before task performance has plateaued. On the other hand, if

717 cardinal biases are only detectable after the model has reached asymptotic performance, this

718 would argue against the idea that they critically contribute to task performance. As shown in

719 Figure 9, our results support the first possibility. For a network trained on upright images,

720 Fisher information profiles measured as early as 50,000 steps into the training process already

721 demonstrated pronounced peaks near the cardinal orientations (Figure 9A; for reference, our

722 main analyses were performed at 400,000 steps). These peaks were not present in the model

723 prior to any training (compare to Figure 2A, gray lines). Interestingly, these peaks in FI were

724 larger in magnitude and shifted further away from the cardinal orientations than those

725 measured at later timesteps (as far as $15^{\circ}$ shifted). To investigate why this was the case, we

726 also analyzed the tuning properties of single units at several steps during training. This revealed

727 that, as in the fully-trained model, network layers analyzed early in training had many narrowly-

728 tuned (high k) units with tuning centers at the cardinal orientations (Figure 9B). However, in

729 addition, there were many units with narrow tuning whose centers lay a few degrees off of the 
730 cardinal orientations (additional "peaks" in the scatter plot in Figure 9B). The presence of these

731 additional, off-cardinal-preferring, units is likely related to the shape of the FI measured at early

732 timesteps. We speculate on this issue further in the Discussion.

734 As a quantitative measure of how cardinal bias evolved over time, we computed the FIB-0 as

735 described in previous sections (Figure 9C, 9D). At all timesteps, FIB-0 tended to increase with

736 depth in the network, as in the fully-trained model. Comparing values across training time, FIB-

7370 was generally maximal at 100,000 steps into the training process. We note, however, that this

738 measure was computed by averaging the $\mathrm{FI}$ within $20^{\circ}$ bins near the cardinals (see Methods,

739 Fisher information bias), and the very shifted peaks we saw at 50,000 steps were outside this

740 window. Thus, this measure does not capture the high degree of non-uniformity we see at

74150,000 steps. With this in mind, the overall amount of bias generally appeared to be highest

742 early in training, before performance began to plateau, and decreased with additional training.

743 At the same time, more training resulted in peaks in FI that were closer to the exact cardinal

744 orientations, reflecting a better match to the prior distribution of orientations in the training

745 set. It is possible that training this model for longer durations or modifying its hyperparameters

746 to reach better plateau performance would result in further shifts in the FI peaks. However, we

747 note that the pre-trained VGG-16 model discussed earlier (Silberman \& Guadarrama, 2016)

748 showed a similarly shaped Fl curve that had peaks around the same orientations as the latest

749 timestep of our model. Thus, it is not likely that the shape we observed is solely dependent on

750 our choice of hyperparameters or training duration. 
A
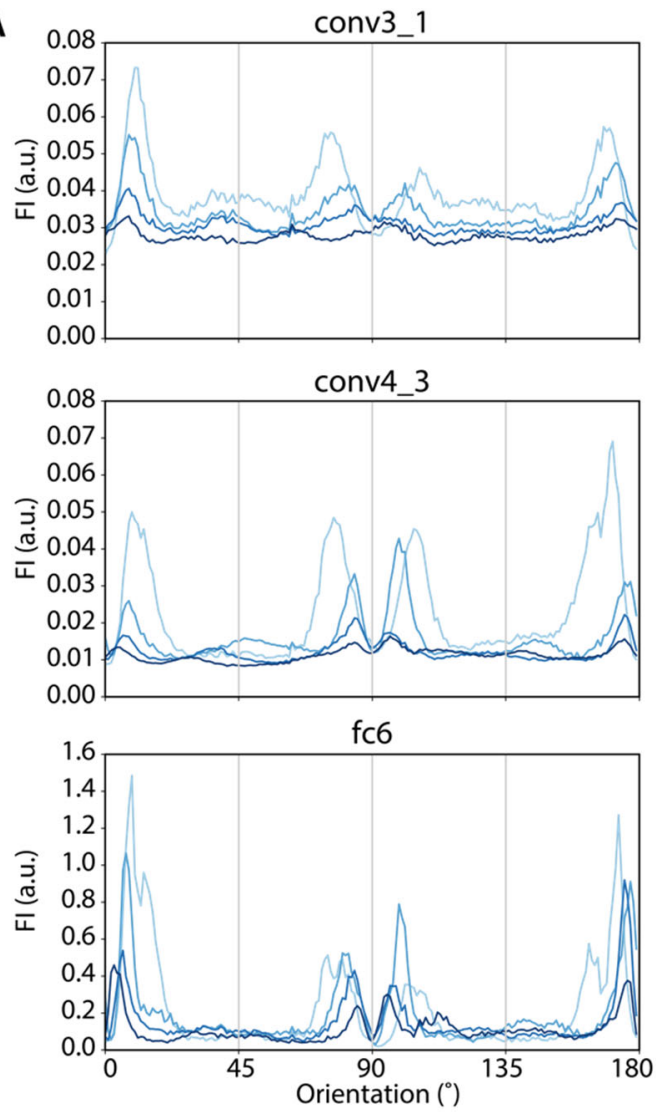

C

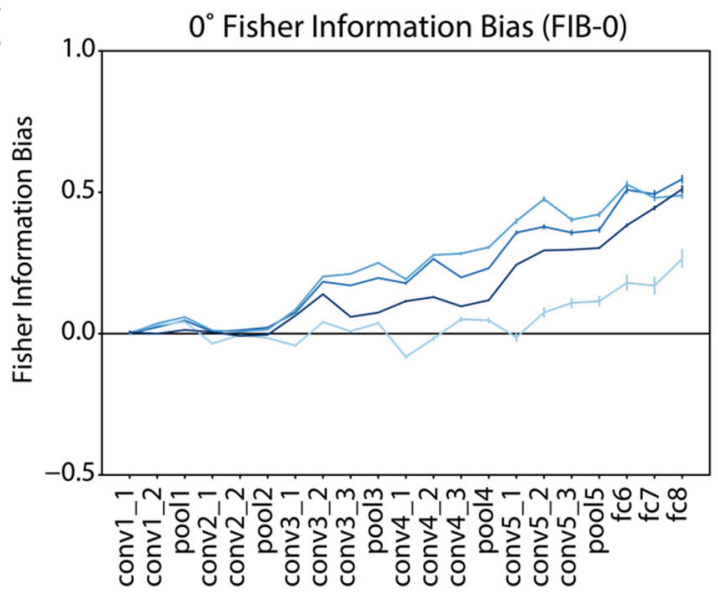

B
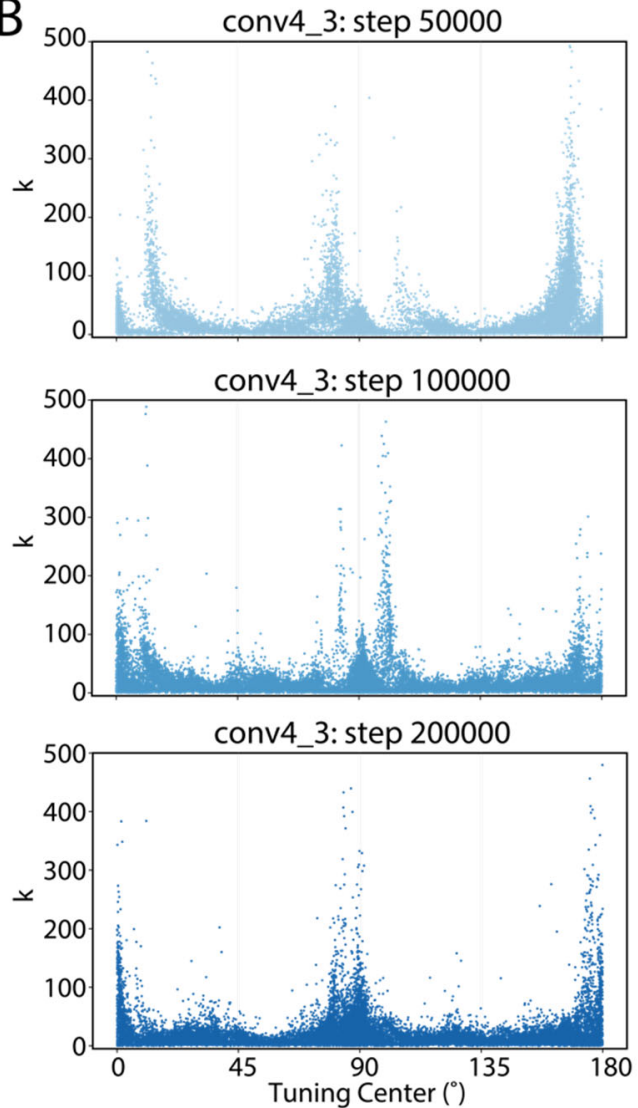

D

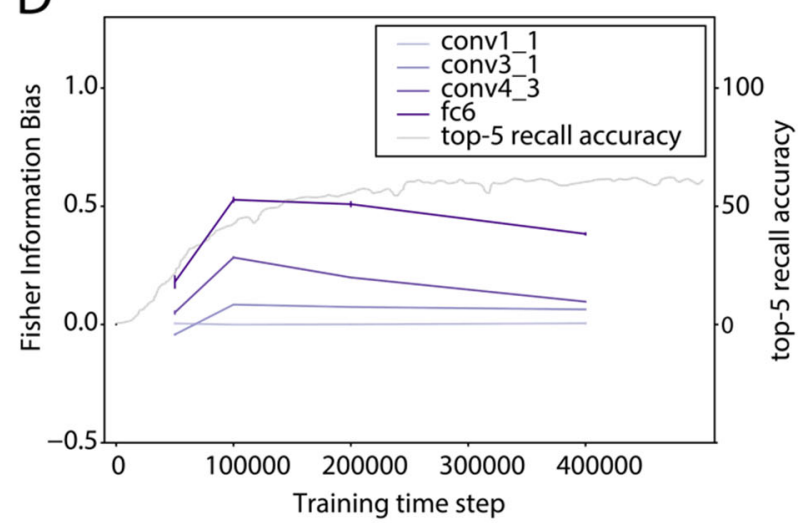

Figure 9. Biases in Fisher information and unit tuning properties over the course of training on

754 upright images. (A) Fisher information for three example layers, at several timepoints during

755 training (shades of blue). For comparison, analyses in Figures 2 and 3 were performed at step

756400,000 (darkest blue line). (B) Concentration parameter (k) versus tuning center, for individual

757 units at conv4_3, plotted for several timepoints during training. Data have been down-sampled 
758 to a maximum of 20,000 points for visualization purposes. (C) FIB-0 across layers plotted for

759 several timepoints (shades of blue). Error bars reflect \pm 1 standard deviation of the measure

760 across 4 evaluation image sets. (D). FIB-0 is plotted as a function of time, for several example

761 layers (purple lines). Light gray line indicates model performance (top-5 recall accuracy), after

762 smoothing with a Gaussian kernel. Error bars reflect \pm 1 standard deviation of the measure

763 across 4 evaluation image sets.

765 Discussion

766

767 We investigated whether CNNs trained to perform object classification exhibit biased

768 orientation representations that reflect non-uniformities in the statistics of the training set

769 images. We found that middle and deep layers of a pre-trained VGG-16 network (Silberman \&

770 Guadarrama, 2016; Simonyan \& Zisserman, 2014) represented orientation with higher

771 discriminability near the cardinal (vertical and horizontal) orientations, with relatively lower

772 discriminability around oblique (diagonal) orientations. Bias was also seen in the tuning

773 properties of single units in the network: there was an over-representation of units that

774 preferred the cardinal orientations, and units tuned to the cardinal orientations had narrower

775 tuning profiles. Furthermore, when we trained models with the same architecture on rotated

776 versions of ImageNet, each of these effects shifted by a predictable amount, such that

777 discriminability was highest near whichever orientations were most common in the network's

778 training set. These results demonstrate that general visual experience with non-uniform image 
779 statistics is sufficient to produce, in a neural network, the same kind of biases that are observed

780 for low-level feature representations in a range of animal species.

781

782 In general, the strength of the biases we measured tended to increase with depth in each

783 network, showing little or no bias in the first 4-6 layers (Figure 3, Figure 4B, Figure 8). In

784 primates, neural correlates of the oblique effect, reflected by an over-representation of

785 cardinal-tuned neurons, have been shown in V1 (Celebrini, Thorpe, Trotter, \& Imbert, 1993; De

786 Valois, William Yund, \& Hepler, 1982; Mansfield, 1974), V2 (Shen et al., 2014), and IT cortex

787 (Vogels \& Orban, 1994). To relate these physiology findings to our results, we can consider a

788 recent finding that for a similar network, VGG-19, the ability of network activations to explain

789 data from primate V1 was best at an intermediate layer, conv3_1, suggesting that earlier layers

790 of the model might be more analogous to processing in the retina and/or lateral geniculate

791 nucleus (Cadena et al., 2019). Therefore, our observation that bias did not emerge until the

792 middle layers of the VGG-16 model is roughly consistent with a cortical origin for the oblique

793 effect. Beyond the middle layers, we found that Fisher information bias (Figure 3, Figure 4B),

794 continued to increase with depth in the network. At the same time, we note that another

795 metric of orientation separability, in which we computed a t-statistic of distances in principal

796 component space (Figure S2), showed similar values of bias at middle and late model layers.

797 Thus, the extent to which biases are magnified versus merely reproduced at later model layers

798 is not entirely clear. In either case, all of the methods that we employed suggest that late layers

799 of the model consistently show strong cardinal biases. This finding is consistent with some 
800 behavioral and physiological results suggesting that the primate oblique effect may be

801 dependent on higher-order processing beyond V1 (Shen et al., 2014; Westheimer, 2003).

802

803 Another property of the biases we observed was that the FI measured in deep layers of each

804 network tended to peak just a few degrees off of the orientations that were most common in

805 the training set, with a dip at the precise locations of the most common orientations (Figure

806 2A). As discussed in the Results, this double-peaked shape follows from the fact that Fl is

807 highest on the flanks of tuning curves, and many narrowly-tuned units in deep layers tended to

808 have their centers around the most common orientations. However, this finding is not generally

809 reflected in human psychophysics, in which the ability to make small orientation discriminations

810 tends to show a single maximum around each of the cardinal orientations (Appelle, 1972;

811 Girshick et al., 2011). One potential reason for this apparent discrepancy is that in this

812 experiment, we were able to present a relatively large number of images (8640 per image set)

813 to the CNN, with images finely spaced by $1^{\circ}$ steps in orientation, whereas psychophysics

814 experiments typically present fewer images at more coarsely spaced orientations (Caelli,

815 Brettel, Rentschler, \& Hilz, 1983; Girshick et al., 2011; Westheimer, 2003). Additionally, we

816 were measuring directly from every unit without any additional sources of downstream noise

817 or interference, which may have made the double-peaked shape of Fisher information more

818 apparent than it would be when estimating orientation thresholds from behavior (Butts \&

819 Goldman, 2006). It is also possible that this qualitative difference between the FI curves we

820 measured and the shape of human discriminability functions represents an actual difference 
821 between visual processing in CNNs and primates. More extensive behavioral experiments may

822 be needed to resolve this.

823

824 From an efficient coding perspective, this double-peaked shape represents a divergence of FI

825 from the shape predicted based on mutual information maximization (Figure 1D; Ganguli \&

826 Simoncelli, 2011; Wei \& Stocker, 2015). This implies that the network is not optimizing based on

827 mutual information but is optimizing another criterion. One interpretation is that rather than

828 being optimized to precisely represent the orientation of stimuli, the network is optimized for

829 simply detecting the most common stimuli, without as much regard to their precise orientation.

830 On this account, the large number of units with their preferred orientations close to the

831 cardinals would serve as effective detectors of cardinally-oriented stimuli but contribute less to

832 the discrimination of these stimuli (Regan \& Beverley, 1985). This interpretation raises the

833 possibility that in the context of object categorization, detecting items with a commonly-

834 encountered orientation is more important than discriminating their orientation.

836 To further investigate the functional role of these biases, we examined the time at which biases

837 emerged during training. In a model we trained on upright images, cardinal biases were

838 detectable early in training, before performance had begun to plateau (Figure 9). This result is

839 consistent with the idea that the biases may contribute to improvements in task performance,

840 rather than emerging only as a late stage byproduct of high performance. Another implication

841 of this result is that the learned features that allow the model to achieve its maximal task

842 performance may not be strictly required for orientation biases to emerge. Rather, it may be 
843 the case that as soon as the network begins to represent a feature such as orientation, the

844 resulting representations exhibit bias. Indeed, the overall measure of cardinal information bias

845 declined from step 100,000 to step 400,000 (Figure 9D), suggesting that biases are initially quite

846 pronounced and become scaled back over the course of training.

847

848 At the same time, the double-peaked shape in FI was even more pronounced early in training,

849 with the peaks further offset from the cardinal axes (Figure 9A). As described earlier in this

850 section, this double-peaked shape may be non-optimal in terms of efficient coding. Thus, it may

851 be the case that training has the dual effects of reducing the magnitude of bias and making the

852 network's representations more closely approximate an efficient encoding of the prior

853 distribution. This process apparently unfolds in parallel to the model's improvement in task

854 performance, leaving open the question of what relationship between these processes is. One

855 interpretation is that optimal orientation coding directly impacts the model's learning process,

856 contributing to its ability to accurately classify objects. Another interpretation is that both the

857 changes in orientation bias and the improvement in object categorization performance are

858 reflections of the same underlying process, in which the network is developing a more accurate,

859 complete representation of the training set distribution. Further experiments will be needed to

860 conclusively resolve this.

861

862 Our findings may also provide some indication of which architectural properties of the VGG-16

863 network are required for cardinal biases to emerge. First, we found that biases tended to

864 emerge around the $5^{\text {th }}$ layer of the model and increase with depth in the network (Figure 3 ). 
865 The fact that there is a minimum depth requirement for biases to emerge may be related to the

866 small convolutional kernels in the VGG-16 model ( $3 \times 3$ pixels), potentially suggesting that a large

867 spatial receptive field is required for the emergence of orientation biases. A potential extension

868 of this work would be to compare our results with another network architecture having a larger

869 convolutional filter size, and examine whether biases are present at a shallower depth in such a

870 model (e.g. AlexNet; Krizhevsky, Sutskever, \& Hinton, 2012). The observation that biases further

871 increase with depth in the model may further suggest that repeated convolutions, spatial

872 pooling operations and fully connected layers result in amplification of the biases. Additionally,

873 more general properties of the convolutional architecture used in VGG-16 might be required for

874 the emergence of the oblique effect. For example, the sharing of weights across units with

875 different spatial selectivity may be a critical feature that enables learning of orientation

876 representations that efficiently encode the training set statistics. The use of a nonlinear

877 activation function may also be key. Manipulating these core aspects of the model is beyond

878 the scope of the present paper, but in future work it would be useful to explore biases in visual

879 representations across a wider range of network architectures. For instance, if it were found

880 that the use of a convolutional architecture, roughly analogous to the architecture of the

881 primate visual system, is critical for cardinal biases to emerge, this might provide insight into

882 the functional relevance of this property in the primate brain.

883

884 Finally, we also observed weak evidence for a cardinal bias in FI measured from the deep layers

885 of a random network with no training (Figure 3, Figure 8A). This may indicate that some aspect

886 of the model's architecture, such as its use of a square image grid, square convolutional kernels, 
887 and pooling operations over square image regions, introduced an intrinsic cardinal reference

888 frame. However, the possible presence of such a reference frame cannot account for the

889 effects we observed for several reasons. First, the magnitude of the FIB-0 was $5 x$ lower for the

890 deepest layer of the random models as compared to the trained-upright models, and the

891 random models did not show an over-representation of cardinal-tuned units, while the upright-

892 trained models did (Figure 2B, Figure 7B, Figure S1). This suggests that the network response

893 properties underlying any intrinsic cardinal Fl bias were different than those underlying the

894 experience-driven biases we observed. Second, the magnitude of the shifted biases we

895 measured in models trained on rotated images were of similar magnitude to the cardinal biases

896 we measured in models trained on upright images (Figure 8), which demonstrates that having

897 an intrinsic reference frame that matches the orientation distribution of training images is not

898 required for a substantial bias to emerge. These results suggest that training may be able to

899 override some intrinsic response properties of CNNs. However, they also highlight the general

900 importance of examining the biases inherent in CNNs before making analogies to the visual

901 system.

902

903 These findings also have general relevance for the use of CNNs in vision research. First, our

904 results show that a popular CNN model exhibits a form of the classical oblique effect,

905 suggesting that this key aspect of low-level primate vision is reproduced by the model. This

906 adds to a growing body of work demonstrating similarities between deep neural networks and

907 the brains and behavior of primates (Kubilius, Bracci, \& Op de Beeck, 2016; Pospisil, Pasupathy,

908 \& Bair, 2018; Rideaux \& Welchman, 2020; Ward, 2019; Yamins et al., 2014). Second, we have 
909 demonstrated that non-uniformities in the statistics of training set images can dramatically

910 influence the feature representations that are learned by a CNN. Specifically, image features

911 that are over-represented during training are likely to be more discriminable by the trained

912 network, which may lead to a performance advantage for processing certain stimuli over

913 others. Accounting for such influences is critical for avoiding unwanted algorithmic biases,

914 particularly in modeling high-level visual functions such as face recognition (Buolamwini \&

915 Gebru, 2018; Cavazos, Phillips, Castillo, \& O’Toole, 2019; Klare, Burge, Klontz, Vorder Bruegge,

916 \& Jain, 2012).

917

918 Overall, our results suggest that the classical oblique effect is reproduced in a CNN trained to

919 perform object recognition on an image set containing an over-representation of cardinal

920 orientations. Furthermore, a rotated version of this bias can be induced by training a CNN on

921 rotated versions of these same images. These results indicate that general visual experience,

922 without the presence of an innate bias that matches the viewed orientation distribution, is

923 sufficient to induce the formation of orientation biases, providing support for an experience-

924 driven account of the oblique effect.

925

926 Acknowledgements

927

928 Funded by NEI R01-EY025872 to JTS, and NIMH Training Grant in Cognitive Neuroscience (T32-

$929 \mathrm{MH020002)}$ to MMH. We thank the San Diego Supercomputer Center and UCSD's Social Sciences 
bioRxiv preprint doi: https://doi.org/10.1101/2020.07.17.209536; this version posted March 30, 2021. The copyright holder for this preprint (which was not certified by peer review) is the author/funder. All rights reserved. No reuse allowed without permission.

930 Computing Facility for computing support, and the NVIDIA corporation for donation of a Quadro

931 P6000 GPU that was used in this research. 


\section{References}

934 Abadi, M., Barham, P., Chen, J., Chen, Z., Davis, A., Dean, J., ... Zheng, X. (2016). TensorFlow: A

935 system for large-scale machine learning. ArXiv. Retrieved from http://arxiv.org/abs/1605.08695

Abbott, L. F., \& Dayan, P. (1999). The effect of correlated variability on the accuracy of a population code. In Neural Computation (Vol. 11). https://doi.org/10.1162/089976699300016827

Appelle, S. (1972). Perception and discrimination as a function of stimulus orientation: The "oblique effect" in man and animals. Psychological Bulletin, 78(4), 266-278. https://doi.org/10.1037/h0033117

Barlow, H. B. (1961). Possible Principles Underlying the Transformations of Sensory Messages. In Sensory Communication (pp. 217-234).

Bauer, J. A., Owens, D. A., Thomas, J., \& Held, R. (1979). Monkeys Show an Oblique Effect.

$$
\text { Perception, 8(3), 247-253. https://doi.org/10.1068/p080247 }
$$

948 Benjamini, Y., \& Yekutieli, D. (2001). The control of the false discovery rate in multiple testing 949 under dependency. Annals of Statistics, Vol. 29, pp. 1165-1188. https://doi.org/10.1214/aos/1013699998

951 Blakemore, C., \& Cooper, G. F. (1970). Development of the brain depends on the visual environment. Nature, 228(5270), 477-478. https://doi.org/10.1038/228477a0

953 Buolamwini, J., \& Gebru, T. (2018). Gender Shades: Intersectional Accuracy Disparities in 
Commercial Gender Classification *. In Proceedings of Machine Learning Research (Vol.

Butts, D. A., \& Goldman, M. S. (2006). Tuning Curves, Neuronal Variability, and Sensory Coding. PLoS Biology, 4(4), e92. https://doi.org/10.1371/journal.pbio.0040092

Cadena, S. A., Denfield, G. H., Walker, E. Y., Gatys, L. A., Tolias, A. S., Bethge, M., \& Ecker, A. S. (2019). Deep convolutional models improve predictions of macaque V1 responses to natural images. PLoS Computational Biology, 15(4), e1006897. https://doi.org/10.1371/journal.pcbi.1006897

Caelli, T., Brettel, H., Rentschler, I., \& Hilz, R. (1983). Discrimination thresholds in the twodimensional spatial frequency domain. Vision Research, 23(2), 129-133. https://doi.org/10.1016/0042-6989(83)90135-9

Cavazos, J. G., Phillips, P. J., Castillo, C. D., \& O’Toole, A. J. (2019). Accuracy comparison across face recognition algorithms: Where are we on measuring race bias? Retrieved from http://arxiv.org/abs/1912.07398

Celebrini, S., Thorpe, S., Trotter, Y., \& Imbert, M. (1993). Dynamics of orientation coding in area VI of the awake primate. Visual Neuroscience, 10(5), 811-825.

971 Cichy, R. M., \& Kaiser, D. (2019). Deep Neural Networks as Scientific Models. Trends in Cognitive Sciences, 23(4), 305-317. https://doi.org/10.1016/j.tics.2019.01.009

973 Coppola, D. M., Purves, H. R., McCoy, A. N., \& Purves, D. (1998). The distribution of oriented 974 contours in the real world. Proceedings of the National Academy of Sciences of the United 975 States of America, 95(7), 4002-4006. https://doi.org/10.1073/pnas.95.7.4002 
976 Coppola, D. M., \& White, L. E. (2004). Visual experience promotes the isotropic representation

977 of orientation preference. Visual Neuroscience, 21(1), 39-51.

978 https://doi.org/10.1017/s0952523804041045

979 De Valois, R. L., William Yund, E., \& Hepler, N. (1982). The orientation and direction selectivity

980 of cells in macaque visual cortex. Vision Research, 22(5), 531-544.

981 https://doi.org/10.1016/0042-6989(82)90112-2

982 Deng, J., Dong, W., Socher, R., Li, L.-J., Kai Li, \& Li Fei-Fei. (2009). ImageNet: A large-scale

983 hierarchical image database. In 2009 IEEE Conference on Computer Vision and Pattern

984 Recognition. https://doi.org/10.1109/CVPRW.2009.5206848

985 Ganguli, D., \& Simoncelli, E. P. (2011). Implicit encoding of prior probabilities in optimal neural

986 population (Vol. 23). Retrieved from MIT Press website: http://www.nips.cc

987 Girshick, A. R., Landy, M. S., \& Simoncelli, E. P. (2011). Cardinal rules: visual orientation

988 perception reflects knowledge of environmental statistics. Nature Neuroscience, 14(7),

989 926-932. https://doi.org/10.1038/nn.2831

990 Hirsch, H. V. B., \& Spinelli, D. N. (1970). Visual experience modifies distribution of horizontally

991 and vertically oriented receptive fields in cats. Science, 168(3933), 869-871.

992 https://doi.org/10.1126/science.168.3933.869

993 Hoy, J. L., \& Niell, C. M. (2015). Layer-specific refinement of visual cortex function after eye

994 opening in the awake mouse. The Journal of Neuroscience: The Official Journal of the

995 Society for Neuroscience, 35(8), 3370-3383. https://doi.org/10.1523/JNEUROSCI.3174-

$996 \quad 14.2015$

997 Jain, A. K., \& Farrokhnia, F. (1991). Unsupervised texture segmentation using Gabor filters. 
Pattern Recognition, 24(12), 1167-1186. https://doi.org/10.1016/0031-3203(91)90143-S

999

1000

1001

1002

1003

1004

1005

1006

1007

1008

1009

1010

1011

1012

1013

1014

1015

1016

1017

1018

1019

Kell, A. J., \& McDermott, J. H. (2019, April 1). Deep neural network models of sensory systems: windows onto the role of task constraints. Current Opinion in Neurobiology, Vol. 55, pp. 121-132. https://doi.org/10.1016/j.conb.2019.02.003

Klare, B. F., Burge, M. J., Klontz, J. C., Vorder Bruegge, R. W., \& Jain, A. K. (2012). Face recognition performance: Role of demographic information. IEEE Transactions on Information Forensics and Security, 7(6), 1789-1801.

https://doi.org/10.1109/TIFS.2012.2214212

Kreile, A. K., Bonhoeffer, T., \& Hübener, M. (2011). Altered visual experience induces instructive changes of orientation preference in mouse visual cortex. Journal of Neuroscience, 31(39), 13911-13920. https://doi.org/10.1523/JNEUROSCI.2143-11.2011

Krizhevsky, A., Sutskever, I., \& Hinton, G. E. (2012). ImageNet Classification with Deep Convolutional Neural Networks. In Advances in Neural Information Processing Systems (Vol. 25). Retrieved from http://code.google.com/p/cuda-convnet/

Kubilius, J., Bracci, S., \& Op de Beeck, H. P. (2016). Deep Neural Networks as a Computational Model for Human Shape Sensitivity. PLOS Computational Biology, 12(4), e1004896. https://doi.org/10.1371/journal.pcbi.1004896

Leventhal, A. G., \& Hirsch, H. V. B. (1975). Cortical effect of early selective exposure to diagonal lines. Science, 190(4217), 902-904. https://doi.org/10.1126/science.1188371

Leventhal, A. G., \& Hirsch, H. V. B. (1980). Receptive-field properties of different classes of neurons in visual cortex of normal and dark-reared cats. Journal of Neurophysiology, 43(4), 1111-1132. https://doi.org/10.1152/jn.1980.43.4.1111 
1020 Li, B., Peterson, M. R., \& Freeman, R. D. (2003). Oblique Effect: A Neural Basis in the Visual Cortex. Journal of Neurophysiology, 90(1), 204-217.

Mansfield, R. J. W. (1974). Neural basis of orientation perception in primate vision. Science, 186(4169), 1133-1135. https://doi.org/10.1126/science.186.4169.1133

Pospisil, D. A., Pasupathy, A., \& Bair, W. (2018). 'Artiphysiology' reveals V4-like shape tuning in a deep network trained for image classification. ELife, 7. https://doi.org/10.7554/eLife.38242

Regan, D., \& Beverley, K. I. (1985). Postadaptation orientation discrimination. Journal of the

Rideaux, R., \& Welchman, A. E. (2020). But still it moves: Static image statistics underlie how we see motion. Journal of Neuroscience, 40(12), 2538-2552. https://doi.org/10.1523/JNEUROSCI.2760-19.2020

Russakovsky, O., Deng, J., Su, H., Krause, J., Satheesh, S., Ma, S., ... Fei-Fei, L. (2015). ImageNet visual area 2 of macaque monkeys. Journal of Vision, 14(2). https://doi.org/10.1167/14.2.3

1039 Silberman, N., \& Guadarrama, S. (2016). TensorFlow-Slim image classification model library.

1040 Simonyan, K., \& Zisserman, A. (2014). Very Deep Convolutional Networks for Large-Scale Image 
1042 Vogels, R., \& Orban, G. A. (1994). Activity of inferior temporal neurons during orientation

1043 discrimination with successively presented gratings. Journal of Neurophysiology, 71(4),

1044 1428-1451. https://doi.org/10.1152/jn.1994.71.4.1428

1045 Wainwright, M. J. (1999). Visual adaptation as optimal information transmission. Vision

1046 Research, 39(23), 3960-3974. https://doi.org/10.1016/S0042-6989(99)00101-7

1047 Ward, E. J. (2019). Exploring perceptual illusions in deep neural networks. Journal of Vision,

1048 19(10), 34b. https://doi.org/10.1167/19.10.34b

1049 Wei, X.-X., \& Stocker, A. A. (2015). A Bayesian observer model constrained by efficient coding

1050 can explain “anti-Bayesian” percepts. Nature Neuroscience, 18(10), 1509-1517.

$1051 \quad$ https://doi.org/10.1038/nn.4105

1052 Westheimer, G. (2003). Meridional anisotropy in visual processing: Implications for the neural

1053 site of the oblique effect. Vision Research, 43(22), 2281-2289.

1054 https://doi.org/10.1016/S0042-6989(03)00360-2

1055 Yamins, D., Hong, H., Cadieu, C. F., Solomon, E. A., Seibert, D., \& DiCarlo, J. J. (2014).

1056 Performance-optimized hierarchical models predict neural responses in higher visual

1057 cortex. Proceedings of the National Academy of Sciences, 111(23), 8619-8624.

1058 https://doi.org/10.1073/pnas.1403112111

1059

1060

1061

1062

1063 


\section{Supplementary Figures}

A Proportion of orientation-tuned units $\left(r^{2}>0.40\right)$

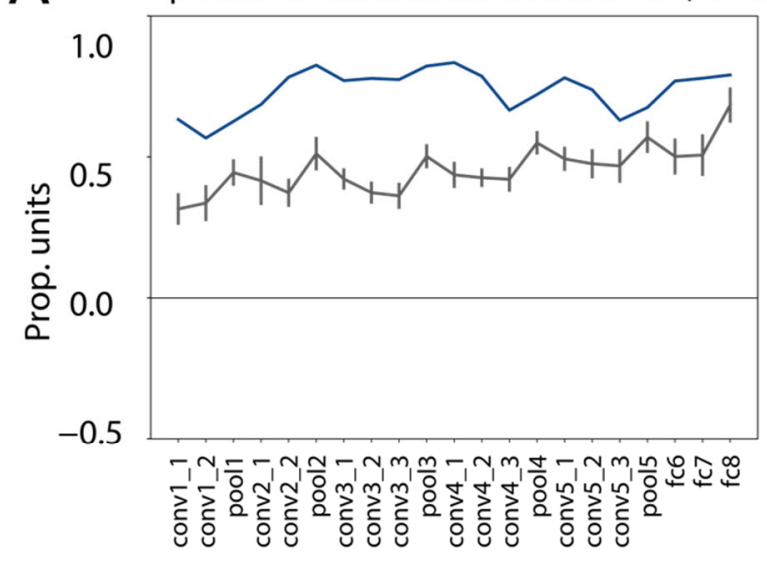

Randomly Initialized VGG-16

Pre-Trained VGG-16

B
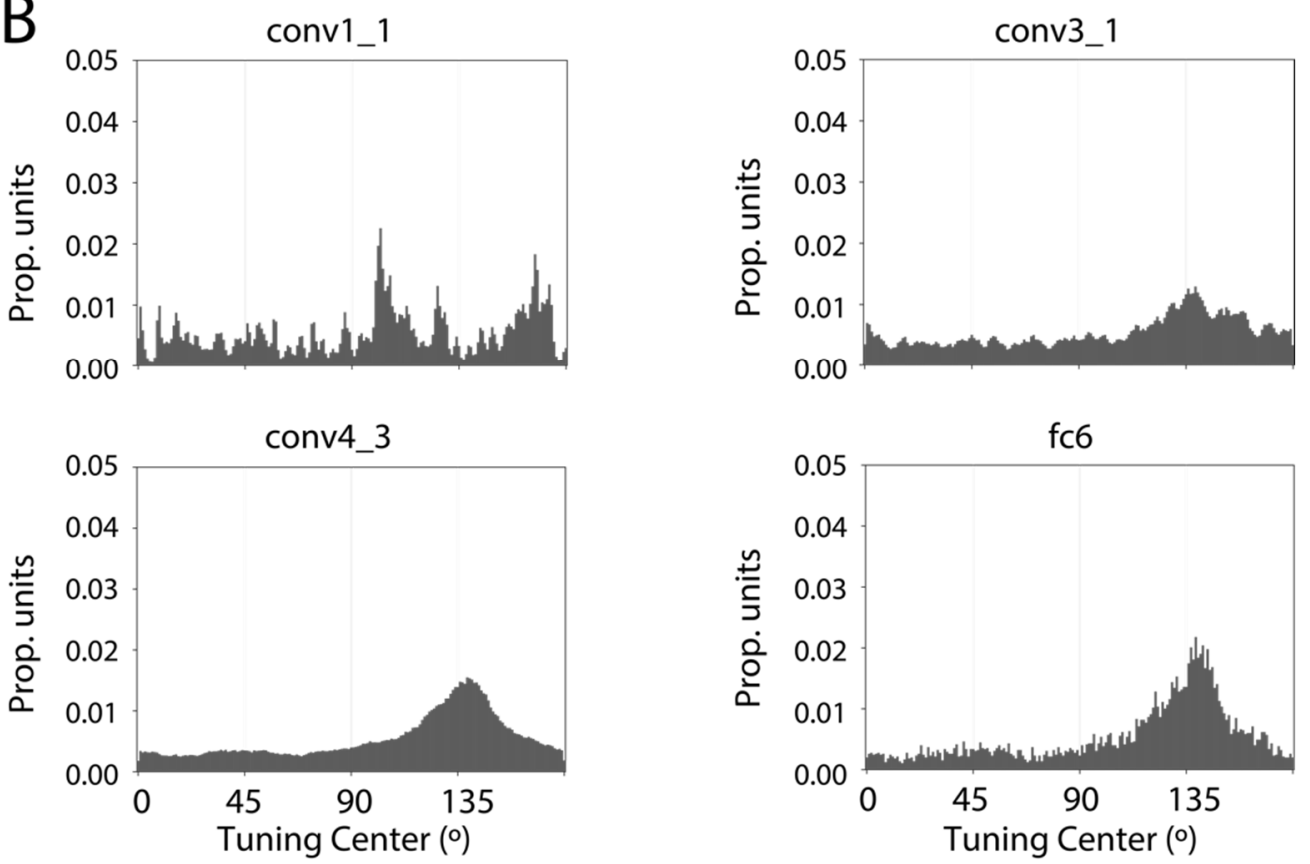

Figure S1. (A) Proportion of units in each layer that were well-fit by a Von Mises function (see

1067 Methods, Single-unit tuning analysis), for a pre-trained VGG-16 model (navy blue) and randomly

1068 initialized models with no training (gray). Error bars on the gray line reflect standard deviation

1069 across four different random initializations of the model. (B) Distribution of unit tuning centers

1070 for the randomly initialized models (distributions are combined across four different random

1071 initializations of the model). 

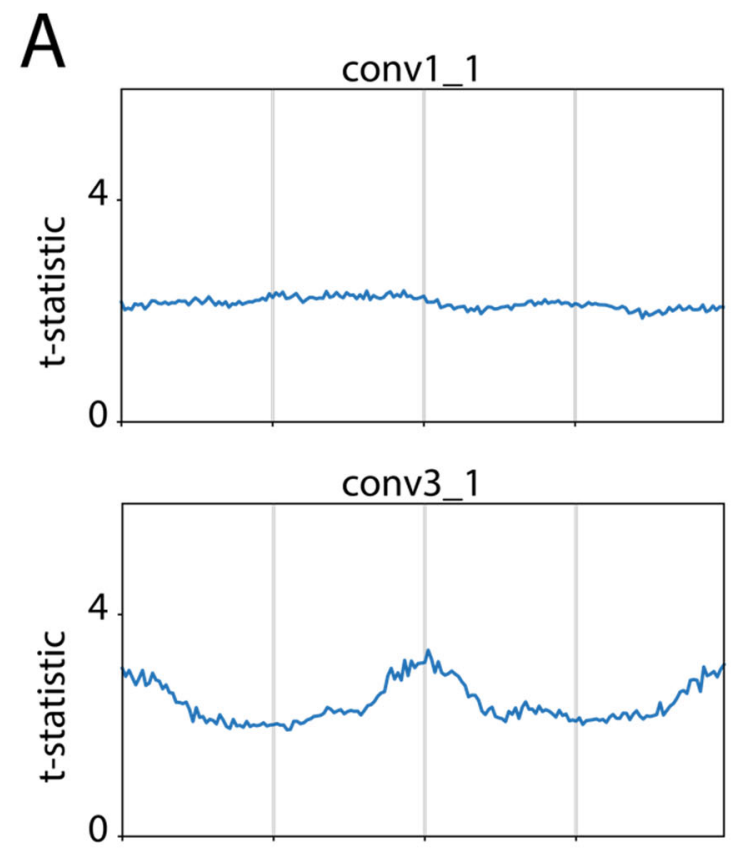

B
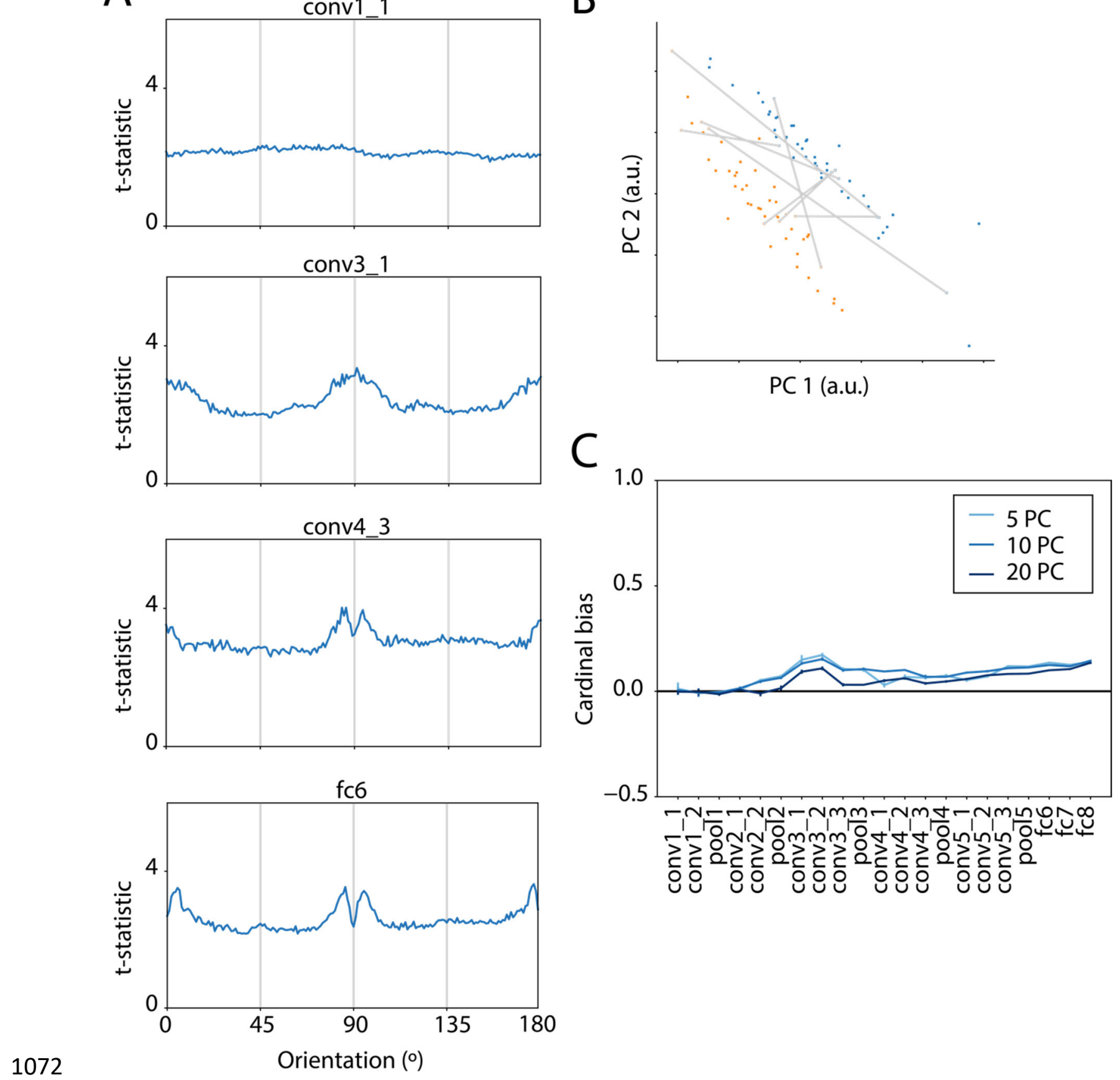

1073 Figure S2. (A) T-statistic for distances between points corresponding to different orientations,

1074 in principal component space. Calculation was done using the first 10 PCs (see Methods,

1075 Multivariate analyses). (B) Schematic of how the t-statistic distance measure was calculated in

1076 PC space: orange and blue dots indicate representations of two nearby orientations, gray lines 
1077 indicate distances between points corresponding to different orientations (note that there are

10782304 such pairwise distances in total; not all distances are drawn). The t-statistic corresponds to

1079 the mean of these distances divided by the standard deviation of the distances. (C) A measure

1080 equivalent to FIB (see Methods, Fisher information bias) for the t-statistic distance is plotted for

1081 each layer, comparing results with 5, 10 or 20 principal components. Error bars reflect \pm 1

1082 standard deviation of the measure across 4 evaluation image sets.

1083 